\title{
Stochastic Growth with Correlated Production Shocks
}

\author{
John B. Donaldson and Rajnish Mehra* \\ Graduate School of Business, Columbia University, \\ New York, New York 10027
}

Received August 13, 1981; revised September 30, 1981

\begin{abstract}
This paper extends the stochastic growth model of Brock and Mirman [J. Econ. Theory 4 (1972), 497-513 | to allow the production shocks to be correlated over time. The resultant optimal savings and consumption policies depend not only upon the current level of output but also upon the most recent realization of the random shock. The properties of these policy functions are studied and it is shown that the Markov process on output, capital stock and consumption resulting from the application of these policies converges to a stationary distribution. Journal of Economic Literature classification numbers: 022, 023, 111, 113.
\end{abstract}

\section{INTRODUCTION}

In a widely referenced paper, Brock and Mirman [5] extend, in the context of the one good neoclassical model, the theory of optimal growth to accommodate uncertainty in the production technology. They show that such a generalized model will behave analogously to the deterministic formulations of Cass [7] and Koopmans [15]; that is, optimal savings and investment policies exist and by their application the economy converges (in an appropriate stochastic sense) to a steady state (distribution). Their random element may be interpreted to capture either observational errors in the measurement of aggregate capital stock or actual uncertainty in the production process (e.g., as crop yield is influenced by uncertain rainfall) for given levels of inputs. By employing a generalized concave technology, Brock and Mirman [5] substantially extend the earlier work of Phelps [26] and Levhari and Srinivasan [17] who employ only linear technologies. Other related work includes that of Mirrlees [25], Hahn [13], Merton [22], Brock and Mirman [6], Mirman and Zilcha [23]. All of the studies mentioned thus

* We would like to gratefully acknowledge the helpful comments of Fischer Black, William A. Brock, V. V. Chari, J. P. Danthine, Edward C. Prescott, and an anonymous referee.

This research was supported by the Faculty Research fund of the Graduate School of Business, Columbia University and the National Science Foundation. 
far require that the random shocks be identically and independently distributed (i.i.d.) over time and their results depend, in a number of ways, crucially upon this assumption.

The classic paper by Lucas and Prescott [19], which characterizes equilibrium in a competitive industry subject to demand uncertainty, was the first to consider correlated shocks. Since they consider an industry equilibrium, however, their analysis is essentially partial equilibrium in nature and certain of their principle results depend heavily upon their specialized production technology. ${ }^{1}$

This paper extends the analysis of Brock and Mirman [5] and Danthine Donaldson [8] to allow the production shocks to be correlated over time. In addition to being a natural generalization of earlier work, such a task better approximates reality, since the introduction of correlated shocks enables us to introduce explicitly the role of expectations. This is due to the fact that the current realization of the random shock will contain information as to the likely future realizations. In our model the optimal savings and consumption policies depend not only upon the current level of output (as, for example, in Brock and Mirman [5,6]) but also upon the most recent realization of the random shock. Expectations, we feel, significantly influence consumption, savings, and investment decisions, and our formulation thus provides a framework in which to examine alternative aggregate savings/consumption behavior arising from different expectations.

Lastly, such expectational dependency allows one to generate, for an appropriate structure on the time path of the random shock, cyclical behavior (persistence) in the aggregate series for consumption, output, and capital stock. Although we leave a thorough discussion of this issue to a future paper, it should be mentioned that our model would allow us to investigate how the characteristics of these cycles (e.g., amplitude) could be influenced by various parameter shifts (such as an increased desire to save as evidenced by an increase in the societal discount factor). Such work would follow the seminal piece of Lucas [18]; adaptations of our techniques, to admit preference uncertainty, would allow us to address issues raised in Black [1]. Thus we regard the work presented here not only as a generalization of the work of Brock and Mirman [5], but also as preliminary to future studies which we feel are likely to be of interest for their policy implications.

An outline of this paper is as follows: In Section II, we formally define the model and derive the existence of the optimal savings/consumption policies. In Section III we further investigate the properties of these policy functions, and, in particular, show that savings (consumption) decrease (increases) as a

\footnotetext{
${ }^{1}$ For example, in the case in which their demand shocks are i.i.d., the asymptotic stationary distribution on firm capital is degenerate (either strictly positive or zero).
} 
proportion of output as output rises. Besides being of interest vis-à-vis macro policy studies, this result replicates the savings behavior actually observed in numerical simulations of various parametric specifications of the model. It has, however, greater significance. Our objective is to show that the Markov processes on output, capital stock, and consumption, resulting from the application of these policies converge to stationary distributions. If output is used as the state variable (i.e., the variable for which convergence is first shown with convergence of the other series following as a corollary from it), standard arguments developed for the i.i.d. shock models (see Danthine and Donaldson [8]) cannot be applied without this additional property. This is due to the dependence of the savings function on the realization of the random shock.

Section IV develops these convergence results. Following Danthine and Donaldson [8], our approach to convergence parallels that suggested by Mendelssohn and Sobel [21], and discussed by Blume [2]. We feel this approach is somewhat simpler than that of Brock and Mirman [5]. The core of these results is Lemma 4.3, which proves that for each realization of the random shock the savings function crosses the $45^{\circ}$ line only once. This enables us to prove that the Markov process on capital stock and output is irreducible on a closed interval. Section $V$ considers the effects of changes in expectations on the optimal policy functions. Lastly, Section VI outlines the future work to be based upon results presented here.

\section{THE ECONOMY}

We consider a modification of the classical model analysed in the stochastic growth literature. ${ }^{2}$ The economy is a specialized (one good) version of that considered in Prescott and Mehra [27]. Society is assumed to consist of infinitely lived individuals with identical preferences and endowments of initial capital stocks and labor (one unit). Since all individuals are alike, the Pareto optimal allocation of interest is the one for which the weights for all individuals' utility functions are equal. To characterize this optimum one can thus either maximize an equal weight aggregate utility function (the social welfare function of the society) or, alternatively, since the assumed strict concavity of the individual utility functions (see below) implies that the optimal decisions of all the individuals would be identical, we can characterize the optimal consumption, savings (investment) program of a representative "stand in" individual. We shall follow the latter convention in this paper.

\footnotetext{
${ }^{2}$ See specially Brock and Mirman $[5,6]$; for an excellent overview of the growth literature see Dixit [10]. For an adaptation to business cycle theory see Kydland and Prescott [16].
} 
There is one capital good. The capital stock per worker available to the economy at time $t,\left(k_{t}\right)$, can be used to produce output which can either be consumed or saved and used for production next period. Production requires both capital and labor and productive units are subject to stochastic shocks over time which affect their productivity. Unlike previous analyses we allow production shocks to be correlated over time.

Each period, a typical consumer chooses a real vector $x_{t} \in R^{4}$, his period $t$ commodity point, from his period $t$ consumption possibility set $X\left(k_{t}\right)$. The vector $x_{t}$ describes the quantities of commodities he actually consumes (positive) and the quantities of inputs (negative) he provides. We find it convenient to split $x_{t}$ into three sets of components $x_{t}=\{(c,-l),(-z), s\}$. The component $z \in R^{1}$ corresponds to the capital supplied by the individual for production, while $(c,-l) \in R^{2}$ corresponds to the individual's period $t$ consumption and supplied labor service. Lastly, $s \in R^{1}$ represents the individual's savings in period $t$ to be used as capital in period $t+1$.

The individual is constrained to choose $x \in X$ in each period. This set is closed and convex and imposes constraints upon the components of $(c,-l)$ such as labor $(l)$ supplied does not exceed available time. In addition the capital supply, $z$, is constrained by the available capital $k$. Thus the period consumption possibility set given $k$ is

$$
\begin{aligned}
X(k) & =\{x: x \in X \text { and }-k \leqslant-z \leqslant 0\} \\
& =\left\{(c,-l,-z, s) \in R^{4} \mid s, c \geqslant 0,0 \leqslant l \leqslant 1, z \leqslant k\right\} .
\end{aligned}
$$

The individual's holdings of capital next period (that is $k_{t+1}$ ) is equal to the amount of output saved this period (that is $s_{t}$ ). Thus $k_{t+1}=s_{t}$ specifies how capital holdings depend upon the previous period's decision. The set of possible capital stocks is the positive real line.

We make the following assumptions (to be maintained throughout the paper) regarding preferences and technology:

A1. It is assumed that the random shock $\lambda \in R^{1}$ is subject to a stationary Markov process with a strictly positive bounded ergodic set $\Lambda$. The transition function of the process is $G: \Lambda \times A \rightarrow[0,1] . G(;)$ is continuous in both its arguments and for fixed $\lambda, G(; \lambda)$ is the probability distribution for the next period's shock. $A=[\underline{\lambda}, \bar{\lambda}], 0<\underline{\lambda}<\bar{\lambda}<\infty$ and $\forall \lambda_{t} \in A$

$$
\begin{array}{ll}
G\left(\lambda_{t+1} ; \lambda_{t}\right)=0, & \lambda_{t+1} \leqslant \underline{\lambda}, \\
G\left(\lambda_{t+1} ; \lambda_{t}\right)=1, & \lambda_{t+1} \geqslant \bar{\lambda} .
\end{array}
$$

Furthermore, we require that the density function $d G(\cdot ; \cdot)$ associated with 
$G(\cdot ; \cdot)$ be continuous on $\Lambda \times \Lambda$, strictly positive $\left(d G\left(\lambda_{1} ; \lambda_{2}\right)>0, \forall \lambda_{1}, \lambda_{2} \in\right.$ $\Lambda \times \Lambda$ ), and that there exists an $L>0$, small, such that $\forall \lambda_{1}, \lambda_{2} \in \Lambda$,

$$
\int_{\Lambda}\left|d G\left(\lambda ; \lambda_{2}\right)-d G\left(\lambda ; \lambda_{1}\right)\right|<L\left|\lambda_{2}-\lambda_{1}\right|
$$

The actual bounds on $L$ will be made more precise in a later section.

All this is simply to require that the density function be "smooth"; that is, if two production shocks today do not differ by very much, then the conditional distributions on next period's shock must be similarly close in the manner indicated.

A2. $G\left(\lambda_{t+1} ; \lambda_{t}^{\prime}\right)$ stochastically dominates $G\left(\lambda_{t+1} ; \lambda_{t}\right)$ in the first degree (Rothschild and Stiglitz [28]) whenever $\lambda_{t}^{\prime}>\lambda_{t}$. This assumption is intended to capture intuitively the notion that it is more likely that tomorrow will be similar to today rather than radically different. It is illustrated by the transition function shown in Fig. 1.

A3. The representative individual orders his preferences over random consumption paths for which $x_{t} \in X\left(k_{t}\right)$ with probability one and $k_{t+1}=s_{t}$ for all $t$ by

$$
E\left\{\sum_{t=0}^{\infty} \beta^{t} u\left(c_{t}\right)\right\}
$$

where $E\{\cdot\}$ is the expectation operator, $\beta$ is the discount factor, and $u(\cdot)$ is the period utility function. The function $u: X \rightarrow R$ is assumed to be strictly increasing, strictly concave in $c$, bounded and thrice continuously differentiable with $u^{\prime}(0)=+\infty, u^{\prime}(\infty)=0$.

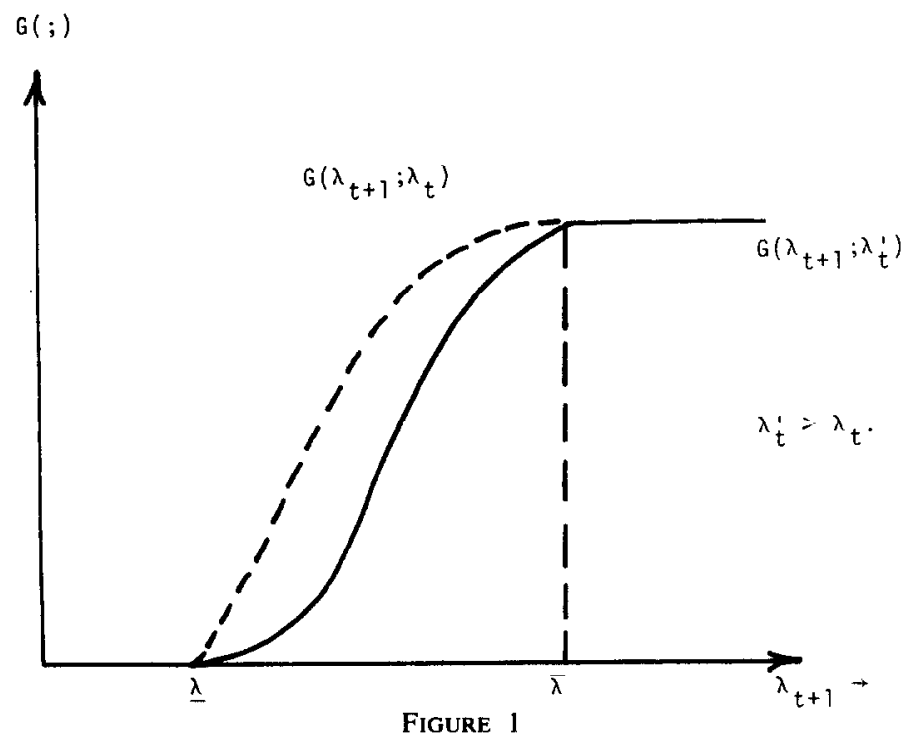


For certain results of ours (Theorem 3.2) we will also require that $u(\cdot)$ display constant or decreasing relative risk aversion, i.e., that $-c u^{\prime \prime}(c) / u^{\prime}(c)$ is constant or decreasing as $c \rightarrow \infty$.

A4. Production takes place under constant returns to scale. This assumption is consistent with percentage growth rates being uncorrelated with firm size (Gibrats Law). The constant returns to scale assumption is innocuous. Typically when the convex constraint set is not a cone, it is because some factor such as land is owned rather than rented by the production unit and is not included in the commodity vector. In general a factor can be added to the commodity vector such that the resulting technology set is a convex cone. (see McKenzie [20]).

Each period an individual chooses a commodity point $y_{t}$ from his production possibility set $Y\left(\lambda_{t}\right)$, where $Y(\lambda)$ is a closed convex set. The commodity vector $y_{t}$ is an element of the same finite dimensional space as $x_{t}$. The constant returns to scale assumption implies that given a random shock $\lambda$ and a positive $\gamma$ if $y \in Y(\lambda)$ then $y y \in Y(\lambda)$.

The production function relating output $(q=c+s)$, input labor $(l)$, and capital $(z)$ is $\lambda l f(z / l)$. The function $f(\cdot)$ is assumed to be increasing, strictly concave, three times continuously differentiable and bounded on $R^{+}$, with $f(0)=0$ and $f^{\prime}(0)=+\infty ; f^{\prime}(\infty)=0$. The production possibility set $Y(\lambda)$ is thus

$$
Y(\lambda)=\left\{\begin{array}{l|l}
(c,-l,-z, s) \in R^{4} & \begin{array}{l}
c, l, z, s \geqslant 0 \\
c+s \leqslant \lambda l f(z / l)
\end{array}
\end{array}\right\}
$$

In this economy all relevant information for decision making is summarized by the current capital stock $(k)$ and the current realization of the random shock $(\lambda)$. Thus $(k, \lambda)$ describes the state of the economy. For the technology considered we observe that there is a 1-1 mapping between output $(q)$ and capital stock $(k)$. Hence the state may alternatively be characterized by $(q, \lambda)$ and $(q, \lambda)$ will be termed the state variables. We observe that the structure of the economy is time invariant and the individual solves a similar problem each period. Hence decision rules can be expressed as a function of the state variables.

Formally the problem facing the individual is to search for an optimal set of decision rules $x(q, \lambda)$ which include a consumption policy $c(q, \lambda)$ and a savings policy $s(q, \lambda)$ which solve the following problem, $(P)$.

$$
\begin{aligned}
V\left(q_{0}, \lambda_{0}\right)=\max E & \left\{\sum_{t=0}^{\infty} \beta^{t} u\left(c_{t}\right)\right\} \\
& \text { subject to } x_{t} \in Y\left(\lambda_{t}\right) \cap X\left(k_{t}\right),
\end{aligned}
$$

$$
k_{t+1}=s_{t} \quad \text { for all } t \text { and } q_{0}, \lambda_{0} \text { given. }
$$


Assumptions 1, 3 and 4 are sufficient to prove, as outlined below, the following theorem.

THEOREM 2.1. Given assumptions $\mathrm{A} 1, \mathrm{~A} 3$ and $\mathrm{A} 4$, there exists a unique solution to $(P)$; that is, a unique, bounded, increasing, concave value function $V(\cdot)$ such that

$$
V(q, \lambda)=\max _{c<q}\left\{u(c)+\beta \int V\left(f(q-c) \lambda^{\prime}, \lambda^{\prime}\right) d G\left(\lambda^{\prime} ; \lambda\right)\right\},
$$

where $V(q, \lambda)$ is the maximum obtainable expected utility for all feasible consumption paths and $\lambda$ ' represents next period's shock. Furthermore, there also exists a unique, bounded, continuously differentiable, increasing function $c(q, \lambda)$ such that

$$
V(q, \lambda)=u(c(q, \lambda))+\beta \int V\left(f(q-c(q, \lambda)) \lambda^{\prime}, \lambda^{\prime}\right) d G\left(\lambda^{\prime} ; \lambda\right) .
$$

Here $c(q, \lambda)$ is the optimal consumption policy in the sense of attaining maximum expected utility. The optimal savings policy $s(q, \lambda)$ is therefore defined by: $s(q, \lambda)=q-c(q, \lambda)$. Lastly, as a result of the concavity of $V()$, a necessary and sufficient characterization of the optimal consumption policy is given by

$$
u^{\prime}(c(q, \lambda))=\beta f^{\prime}(q-c(q, \lambda)) \int V^{\prime}\left(f(q-c(q, \lambda)) \lambda^{\prime}, \lambda^{\prime}\right) \lambda^{\prime} d G\left(\lambda^{\prime} ; \lambda\right)
$$

or by the envelope theorem

$$
u^{\prime}(c(q, \lambda))=\beta f^{\prime}(q-c(q, \lambda)) \int u^{\prime}\left(c\left(f(q-c(q, \lambda)) \lambda^{\prime}, \lambda^{\prime}\right) \lambda^{\prime} d G\left(\lambda^{\prime} ; \lambda\right) .\right.
$$

Proof. The existence and boundedness of the value function $V$ and the optimal policy functions $c(q, \lambda)$ and $s(q, \lambda)$ as well as the concavity of $V$ as a function of its first argument follows from Prescott and Mehra [27]. The differentiability of $V$ can be shown in the manner of Harris [14]. Finally, assumptions A1, A3 and A4 are sufficient to guarantee that assumptions 1 through 6 and 10 of Blume et al. [3] are satisfied. Hence by the application of their Theorem 3.1 we can conclude that the policy functions $c(q, \lambda)$ and $s(q, \lambda)$ are continuously differentiable.

The economy considered in this section satisfies all the assumptions of Prescott and Mehra [27]. By their analysis, we know that an optimal allocation can be supported as a competitive equilibrium. The salient feature of this result, from our viewpoint is that it allows us to conclude that the 
optimal policies of problem (P) are also the aggregative policies of a decentralized economy in recursive competitive equilibrium. This allows us to interpret our results outside the strict domain of a centrally planned economy.

\section{Policy Functions: Properties}

In this section we investigate further properties of the optimal savings/ consumption policies. Besides being of interest in their own right, these results will also be important for subsequent convergence arguments.

The nature of savings dependence on the random shock realization is first considered. Such dependence is to be expected as the current shock realization provides information as to the distribution of future output states. As will be apparent, however, this information may alter savings behavior in different ways according to the representative agent's degree of risk aversion.

Under our assumptions on the structure of the random shock transition density, if the representative agent is "sufficiently" risk averse it is found that the greater the value of the current shock, the more that is consumed and the less that is saved from a fixed output level. Conversely, an agent who is less risk averse, or who "tends to risk neutrality" will display the opposite behavior: the greater the current shock, the greater the savings and the less the consumption, again from a fixed output level.

To see why this is so, first recall that if we define the sequence of approximating value functions $\left\{V^{n}\left(q, \lambda_{t}\right)\right\}$ recursively by

$$
V^{n}\left(q, \lambda_{t}\right)=\max _{c \leqslant q}\left\{u(c)+\beta \int V^{n-1}\left(f(q-c) \lambda_{t+1}, \lambda_{t+1}\right) d G\left(\lambda_{t+1} ; \lambda_{1}\right)\right\},
$$

and if $c^{n}\left(q, \lambda_{t}\right)$ is the optimal solution for $V^{n}\left(q, \lambda_{t}\right)$, then $c^{n}\left(q, \lambda_{t}\right)$ converges pointwise to $c\left(q, \lambda_{t}\right)$, where $c\left(q, \lambda_{t}\right)$ solves $V\left(q, \lambda_{t}\right)$ (cf. Eq. (1)). Thus certain properties, if displayed for all $c^{n}\left(q, \lambda_{t}\right), n$ sufficiently large, will be inherited by the optimal policy function $c\left(q, \lambda_{t}\right)$. In this light we have Theorem 3.1:

THEOREM 3.1. For all $q \geqslant 0, \quad \lambda \leqslant \lambda_{t} \leqslant \bar{\lambda}, \quad \partial c\left(q, \lambda_{t}\right) / \partial \lambda_{t}>0 \quad(<0)$ (equivalently $\left.\partial s\left(q, \lambda_{t}\right) / \partial \lambda_{t}<0(>0)\right)$ provided, for some $N$, and all $n \geqslant N$, $\partial V_{1}^{n-1}\left(f\left(q-c_{n}\right) \lambda, \lambda\right) \lambda / \partial \lambda<0(>0){ }^{3}$

Proof. For every $n, c^{n}=c^{n}\left(q, \lambda_{t}\right)$ satisfies the following equation:

$$
u^{\prime}\left(c^{n}\right)=\beta f^{\prime}\left(q-c^{n}\right) \int V_{1}^{n-1}\left(f\left(q-c^{n}\right) \lambda_{t+1}, \lambda_{t+1}\right) \lambda_{t+1} d G\left(\lambda_{t+1} ; \lambda_{t}\right) .
$$

${ }^{3}$ The existence of the derivative $\partial c\left(q, \lambda_{t}\right) / \partial \lambda_{t}$ is ensured by Theorem 2.1 . 
Differentiating with respect to $\lambda_{t}$ yields

$$
\begin{aligned}
& u^{\prime \prime}\left(c^{n}\right) \frac{\partial c^{n}}{\partial \lambda_{t}} \\
& =\beta f^{\prime \prime}\left(q-c^{n}\right)\left(\left(-\frac{\partial c^{n}}{\partial \lambda_{t}}\right) \int V_{1}^{n-1}\left(f\left(q-c^{n}\right) \lambda_{t+1}, \lambda_{t+1}\right) \lambda_{t+1} d G\left(\lambda_{t+1} ; \lambda_{t}\right)\right. \\
& \left.\quad+\beta \mid f^{\prime}\left(q-c^{n}\right)\right]^{2}\left(-\frac{\partial c^{n}}{\partial \lambda_{t}}\right) \int V_{11}^{n-1}\left(f\left(q-c^{n}\right) \lambda_{t+1}, \lambda_{t+1}\right) \lambda_{t+1}^{2} d G\left(\lambda_{t+1} ; \lambda_{t}\right) \\
& \quad+\lim _{\varepsilon \rightarrow 0}(\beta / \varepsilon) f^{\prime}\left(q-c^{n}\right)\left[\int\left\{V_{1}^{n-1}\left(f\left(q-c^{n}\right) \lambda_{t+1}, \lambda_{t+1}\right) \lambda_{t+1}\right\}\right. \\
& \left.\quad \cdot d G\left(\lambda_{t+1} ; \lambda_{t}+\varepsilon\right)-d G\left(\lambda_{t+1} ; \lambda_{t}\right)\right] .
\end{aligned}
$$

The sign of this latter limit (which exists by assumptions A.1, A.2, and A.3) clearly determines the sign of $\partial c^{n}\left(q, \lambda_{t}\right) / \partial \lambda_{t}$. Furthermore, the limit will be negative (and thus $\left.\partial c^{n}\left(q, \lambda_{t}\right) / \partial \lambda_{t}>0\right)$ provided $\partial V_{1}^{n-1}\left(f\left(q-c^{n}\right) \lambda_{t+1}, \lambda_{t+1}\right)$ $\lambda_{t+1} / \partial \lambda_{t+1}<0$, and conversely.

The behavior of the consumption/savings policies thus rests on the nature of the dependence of the family of functions $\left\{V_{1}^{n}(f(k), \lambda, \lambda) \lambda\right\}$ on $\lambda$. In particular, if $\partial V_{1}^{n}(f(k) \lambda, \lambda) \lambda / \partial \lambda \leqslant 0$ for $n \geqslant N$, then $\partial c\left(q, \lambda_{t}\right) / \partial \lambda_{t} \geqslant 0$ $\left(\partial d\left(q, \lambda_{t}\right) / \partial \lambda_{t} \leqslant 0\right)$. To see how this result is related to the agent's relative risk aversion, consider the case of $V^{1}(f(k) \lambda, \lambda) \lambda$ : since $V_{1}^{1}(f(k) \lambda, \lambda)=$ $u^{\prime}(f(k) \lambda)$,

$$
\frac{\partial V_{1}^{1}(f(k) \lambda, \lambda) \lambda}{\partial \lambda}=u^{\prime \prime}(f(k) \lambda) f(k) \lambda+u^{\prime}(f(k) \lambda) \leqslant 0,
$$

for all $k \geqslant 0$ if and only if $u^{\prime \prime}(c) c / u^{\prime}(c)<-1$, for all $c \geqslant 0$; that is, if $u()$ displays a "sufficiently" negative degree of relative risk aversion. By the construction of the $V^{n}\left(q, \lambda_{t}\right)$ functions, the degree to which $V_{1}^{1}(f(k) \lambda, \lambda) \lambda$ rises or falls as a function of $\lambda$ substantially imposes the same behavior on $V_{1}^{n}(f(k) \lambda, \lambda) \lambda$, for all $n$. In general, the precise relationship is complex, but the essential point is that if $u(c)$ displays sufficiently negative relative risk aversion, the negativity of $\partial V_{1}^{1}(f(k) \lambda, \lambda) \lambda / \partial \lambda$ will be inherited by $\left\{V_{1}^{n}(f(k) \lambda, \lambda) \lambda\right\}$ for all $n \geqslant 0$.

Theorem 3.1 is dramatically illustrated for the family of preferences defined by $u(c)=\left(c^{y}-1\right) / \gamma$. Indeed, for $\gamma<0$ (corresponding to $\left.u^{\prime \prime}(c) c / u^{\prime}(c)<-1\right)$, it can be shown that $\partial c\left(q, \lambda_{t}\right) / \partial \lambda_{t} \geqslant 0 \forall q, \lambda_{t}$; similarly, for $\gamma>0 \quad\left(u^{\prime \prime}(c) c / u^{\prime}(c)>-1\right), \partial c\left(q, \lambda_{t}\right) / \partial \lambda_{t} \leqslant 0, \forall q, \lambda_{t}$. For the $\gamma=0$ $(u(c)=\log c)$ case, the optimal savings/consumption policies depend only upon current output, ignoring the shock realization value altogether. 
All this enjoys an appealing intuitive explanation. Our results show that highly risk averse agents will effect savings policies which tend to stabilize output and thus consumption. Knowing that a low shock realization is likely (for large shock correlation) to be followed by one similarly low, a highly risk averse agent saves more the lower the shock, thereby hoping to moderate any further potential output (and thus consumption) decline next period. An analogous argument applies when the current shock is high. Less risk averse agents will display the opposite sort of behavior.

While we have been unable to prove, simply, the concavity (convexity) of the savings (consumption) function our second result demonstrates that, for fixed $\lambda$, the proportion of output going to savings (consumption) falls (rises) as the level of output increases. This property is empirically observed and follows principally as a consequence of our next lemma. ${ }^{4}$

Lemma 3.1. Let $p(\gamma) \geqslant 0, h(\gamma) \geqslant 0$ be twice continuously differentiable functions on $(0, \infty)$ for which $h^{\prime}(\gamma)<p^{\prime}(\gamma)<0$ and $\lim _{\gamma \mapsto 0} h(\gamma)=$ $\lim _{\gamma \mapsto 0} p(\gamma)=\infty ; \quad \lim _{\gamma \mapsto \infty} p(\gamma)=\lim _{\gamma \mapsto \infty} h(\gamma)=0$. Suppose also that $p(\gamma) / \gamma p^{\prime}(\gamma)$ is either constant or monotone decreasing as $\gamma \mapsto \infty$ and that for any interval $(\tilde{\varepsilon}, M), \tilde{\varepsilon}>0, M<\infty$, and any $\gamma_{1}, \gamma_{2} \in(\tilde{\varepsilon}, M)$, there is an $N$ for which

$$
\left|\frac{h^{\prime}\left(\gamma_{1}\right)}{p^{\prime}\left(\gamma_{1}\right)}\right|>N \quad \text { and } \quad \frac{N-1}{M}>\frac{p^{\prime \prime}\left(\gamma_{1}\right)}{\left|p^{\prime}\left(\gamma_{2}\right)\right|}
$$

Then for any interval $(\varepsilon, M), \varepsilon>0$, if $a(\gamma) \geqslant 0, b(\gamma) \geqslant 0$ are differentiable functions defined on $(\varepsilon, M)$ which simultaneously satisfy the equations

$$
p(a(\gamma))=h(b(\gamma)) \quad \text { and } \quad a(\gamma)+b(\gamma)=\gamma,
$$

we may conclude $(a(\gamma) / \gamma) \uparrow$ as $\gamma \uparrow$ and $(b(\gamma) / \gamma) \downarrow$ as $\gamma \uparrow$ on $(\varepsilon, M)$.

Remark. The $a(\gamma)$ and $b(\gamma)$ of the lemma will subsequently (Theorem 3.2) be identified with $c(\gamma, \lambda)$ and $s(\gamma, \lambda)$, respectively. The idea of the proof is to relate the desired properties of $a(\gamma)$ and $b(\gamma)$ to properties of the inverse functions of $p()$ and $h()$.

Proof. Let the interval $(\varepsilon, M)$ be given. To show that $(a(\gamma) / \gamma) \uparrow$ as $\gamma \uparrow$ (equivalently, $(b(\gamma) / \gamma) \downarrow$ as $\gamma \uparrow)$ on $(\varepsilon, M)$, it is sufficient to demonstrate that $a^{\prime}(\gamma) / a(\gamma)>b^{\prime}(\gamma) / b(\gamma), \gamma \in(\varepsilon, M)$. To obtain this latter conclusion, we examine the inverse functions of $p($ ) and $h()$ denoted, respectively, by $\hat{p}(y)$ and $\hat{h}(y)$. By the inverse function theorem these functions are defined and continuously differentiable on $(0, \infty)$; by definition, they satisfy $p(p(\gamma))=\gamma$

\footnotetext{
${ }^{4}$ By empirically we mean that numerical solutions to problem (P) always exhibit savings functions with this property.
} 
and $h(h(\gamma))=\gamma$ for all $\gamma$ in $(0, \infty)$. With $p(\gamma)>0, p^{\prime}(\gamma)<0$, and $p(\gamma) / \gamma p^{\prime}(\gamma)$ constant or decreasing, it must be that $1 / \gamma p^{\prime}(\gamma)$ decreases as $\gamma \mapsto \infty$. Since for any $y \in(0, \infty), y=p(\gamma)$ for some $\gamma \in(0, \infty)$, this is equivalent to asserting that $\hat{p}^{\prime}(y) / \hat{p}(y)$ decreases as $y \mapsto 0\left(\hat{p}^{\prime}(y) / \hat{p}(y)=1 / \gamma p^{\prime}(\gamma)\right.$, where $y=p(\gamma))$. By definition of the inverse function, $\forall \gamma$ in $(0, \infty)$,

$$
\hat{p}^{\prime}(p(\gamma)) p^{\prime}(\gamma)=\hat{h}^{\prime}(h(\gamma)) h^{\prime}(\gamma) ;
$$

equivalently,

$$
\frac{\hat{p}^{\prime}(p(\gamma)) p^{\prime}(\gamma)}{\hat{p}(p(\gamma))}=\frac{\hat{h}^{\prime}(h(\gamma)) h^{\prime}(\gamma)}{\hat{h}(h(\gamma))}
$$

But as $h^{\prime}(\gamma)<p^{\prime}(\gamma)<0$ on $(0, \infty)$, we may conclude

$$
\frac{\hat{p}^{\prime}(p(\gamma))}{\hat{p}(p(\gamma))}<\frac{\hat{h}^{\prime}(h(\gamma))}{\hat{h}(h(\gamma))} \quad \text { on that interval. }
$$

There are three cases to be considered: (i) $p(\gamma)>h(\gamma)$ for all $\gamma$ in $(0, M)$; (ii) there exists a $\gamma_{0}$ in $(0, M)$ such that $p\left(\gamma_{0}\right)=h\left(\gamma_{0}\right)$ for which $p(\gamma)>h(\gamma)$ for $\gamma>\gamma_{0}$ and $p(\gamma)<h(\gamma)$ for $\gamma<\gamma_{0}$ (see Fig. 2); and (iii) $p(\gamma)<h(\gamma)$ for all $\gamma$ in $(0, M)$.

We first consider case (i). Since $p(\gamma)>h(\gamma)>0$ on $(0, M)$ and $\hat{p}^{\prime}(y) / \hat{p}(y)$ decreases as $y$ decreases,

$$
\frac{\hat{p}^{\prime}(h(\gamma))}{\hat{p}(h(\gamma))}<\frac{\hat{h}^{\prime}(h(\gamma))}{\hat{h}(h(\gamma))} \quad \text { for } \quad \gamma \in(0, M) .
$$

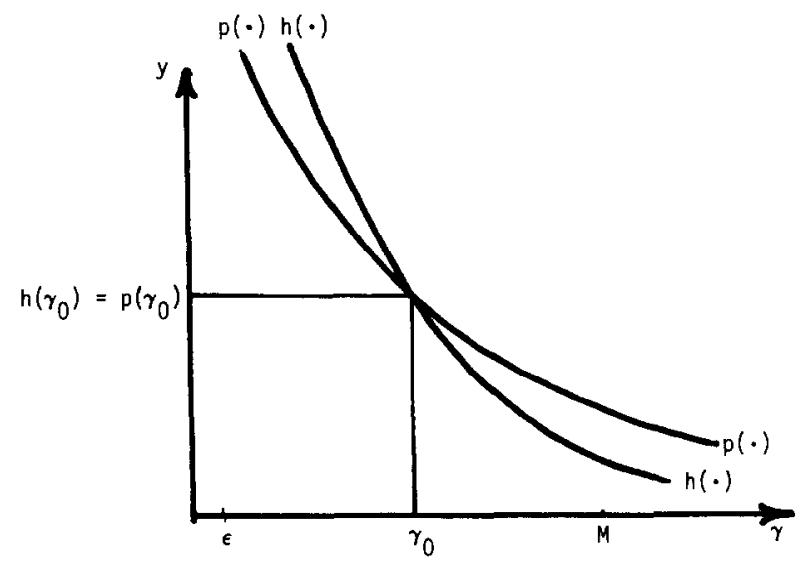

FIGURE 2 
As $\hat{p}(y), \hat{h}(y)$ are continuously differentiable, and as $\lim _{Y \mapsto \infty}(\hat{p}(y)+\hat{h}(y))=0$, for any $\gamma$ in $(\varepsilon, M)$ there must exist a $\hat{y}=\hat{y}(\gamma)$, differentiable, for which $\hat{p}(\hat{y}(\gamma))+\hat{h}(\hat{y}(\gamma))=\gamma$; define $a(\gamma), b(\gamma)$, therefore, by $\hat{p}(\hat{y}(\gamma))=a(\gamma)$ and $\hat{h}(\hat{y}(\gamma))=b(\gamma)$. Differentiating, we have

$$
\hat{p}^{\prime}(\hat{y}(\gamma)) \hat{y}^{\prime}(\gamma)=a^{\prime}(\gamma) \quad \text { and } \quad \hat{h}^{\prime}(\hat{y}(\gamma)) \hat{y}^{\prime}(\gamma)=b^{\prime}(\gamma) .
$$

Since $\hat{p}^{\prime}(y) / \hat{p}(y)<\hat{h}^{\prime}(y) / \hat{h}(y)$ and since $\hat{y}^{\prime}(\gamma)<0$,

$$
\frac{\hat{p}^{\prime}(\hat{y}(\gamma)) \hat{y}^{\prime}(\gamma)}{\hat{p}(\hat{y}(\gamma))}>\frac{\hat{h}^{\prime}(\hat{y}(\gamma)) \hat{y}^{\prime}(\gamma)}{\hat{h}(\hat{y}(\gamma))} .
$$

Equivalently, $a^{\prime}(\gamma) / a(\gamma)>b^{\prime}(\gamma) / b(\gamma)$, for $\gamma \in(\varepsilon, M)$ as desired and case (i) is complete.

For case (ii), the above analysis can be immediately adapted to consider the subcase for which $p(\gamma)>h(\gamma), \gamma>\gamma_{0}$ and thus gives our desired conclusion for the subinterval $\left(2 \gamma_{0}, M\right)$; if $2 \gamma_{0}<\varepsilon$ we are done as well. If not, we next consider the region $\left(0, \gamma_{0}\right)$ on which $p(\gamma)<h(\gamma)$. By continuity, there exists a $\tilde{y}$ for which $\hat{p}(\tilde{y})+\hat{h}(\tilde{y})=\varepsilon$, and consider the interval $(\tilde{\varepsilon}, M)$, where $\tilde{\varepsilon}=\hat{p}(\tilde{y})$. Let $N$ be specified for this interval $(\tilde{\varepsilon}, M)$. By assumption

$$
(N-1)>\frac{p^{\prime \prime}\left(\gamma_{1}\right)}{\left|p^{\prime}\left(\gamma_{2}\right)\right|} M, \quad \forall \gamma_{1}, \gamma_{2} \in(\tilde{\varepsilon}, M) .
$$

But for any $y \leqslant \tilde{y}$ for which $\hat{p}(y), \hat{h}(y)<\gamma_{0}, M>|\hat{p}(y)-\hat{h}(y)|$. Thus for any $\gamma_{1}, \gamma_{2} \in(\tilde{\varepsilon}, M), y \leqslant \tilde{y}$ such that $\hat{p}(y)<\gamma_{0}$

$$
N>1+\frac{p^{\prime \prime}\left(\gamma_{1}\right)}{\left|p^{\prime}\left(\gamma_{2}\right)\right|}|\hat{p}(y)-\hat{h}(y)|
$$

Furthermore, by assumption

$$
\left|\frac{h^{\prime}\left(\gamma_{2}\right)}{p^{\prime}\left(\gamma_{2}\right)}\right|>1+\frac{p^{\prime \prime}\left(\gamma_{1}\right)}{\left|p^{\prime}\left(\gamma_{2}\right)\right|}|\hat{p}(y)-\hat{h}(y)|
$$

or

$$
\left|h^{\prime}\left(\gamma_{2}\right)\right|>\left|p^{\prime}\left(\gamma_{2}\right)\right|+p^{\prime \prime}\left(\gamma_{1}\right)|\hat{p}(y)-\hat{h}(y)| .
$$

Since $\gamma_{2}$ is arbitrary in $(\tilde{\varepsilon}, M)$, we could as well write

$$
\left|h^{\prime}(\hat{h}(y))\right|>\left|p^{\prime}(\hat{h}(y))\right|+p^{\prime \prime}\left(\gamma_{1}\right)|\hat{p}(y)-\hat{h}(y)| .
$$

By the mean value theorem we know there is a $\hat{\gamma}_{1} \in(\tilde{\varepsilon}, M)$ for which, for any $y, y \leqslant \tilde{y}, \hat{p}(y)<\gamma_{0}$,

$$
\left|p^{\prime}(\hat{p}(y))\right|=\left|p^{\prime}(\hat{h}(y))\right|+p^{\prime \prime}\left(\hat{\gamma}_{1}\right)|\hat{p}(y)-\hat{h}(y)| .
$$


But, since $\gamma_{1}$ is arbitrary, we may also write

$$
\left|h^{\prime}(\hat{h}(y))\right|>\left|p^{\prime}(\hat{h}(y))\right|+p^{\prime \prime}\left(\hat{\gamma}_{1}\right)|\hat{p}(y)-\hat{h}(y)|,
$$

from which we may conclude

or

$$
\left|h^{\prime}(\hat{h}(y))\right|>\left|p^{\prime}(\hat{p}(y))\right|
$$

$$
h^{\prime}(\hat{h}(y))<p^{\prime}(\hat{p}(y))
$$

and thus

$$
\hat{p}^{\prime}(y)<\hat{h}^{\prime}(y) \quad \text { for } \quad y \in\left(p\left(\gamma_{0}\right), \tilde{y}\right) .
$$

But this implies

$$
\frac{\hat{p}^{\prime}(y)}{\hat{p}(y)}<\frac{\hat{h}^{\prime}(y)}{\hat{h}(y)}
$$

for all $y$ for which $\gamma>\hat{p}(y)>\tilde{\varepsilon}$ and $\gamma>\hat{h}(y)>\tilde{\varepsilon}$, and our result follows.

Case (ii) follows analogously.

THEOREM 3.2. Let $f^{\prime \prime}(\gamma)<u^{\prime \prime}(\gamma)<0$ on $(0, \infty)$ and suppose $u()$ displays constant or declining relative risk aversion. Let $M$ be some number, $0<M<\sup _{z} f(z) \bar{\lambda}$. Suppose also that for any $\varepsilon, 0<\varepsilon<M$, there exists an $N$ for which

$$
\frac{N-1}{M}>\frac{u^{\prime \prime \prime}\left(\gamma_{1}\right)}{\left|u^{\prime \prime}\left(\gamma_{2}\right)\right|}, \quad \forall \gamma_{1}, \gamma_{2} \quad \text { in } \quad(\varepsilon, M) .
$$

Then, $\forall \lambda_{t},\left(s\left(q, \lambda_{t}\right) / q\right) \downarrow$ as $q \uparrow$, and $\left(c\left(q, \lambda_{t}\right) / q\right) \uparrow$ as $q \uparrow$, for $q \in(\varepsilon, M)$.

Proof. For arbitrary fixed $\lambda_{t}$, the optimal savings and consumption policies satisfy the familiar first order condition

$$
u^{\prime}\left(c\left(q, \lambda_{t}\right)\right)=\beta f^{\prime}\left(s\left(q, \lambda_{t}\right)\right) \int u^{\prime}\left(c\left(f\left(s\left(q, \lambda_{t}\right)\right) \lambda_{t+1}, \lambda_{t+1}\right)\right) \lambda_{t+1} d G\left(\lambda_{t+1} ; \lambda_{t}\right) .
$$

By construction, $s\left(q, \lambda_{t}\right)+c\left(q, \lambda_{t}\right)=q, \forall\left(q, \lambda_{t}\right)$, with $s\left(q, \lambda_{t}\right), c\left(q, \lambda_{t}\right)$ both increasing functions of $q$. Thus $\int u^{\prime}\left(c\left(f\left(s\left(q, \lambda_{t}\right)\right) \lambda_{t+1}, \lambda_{t+1}\right)\right) \lambda_{t+1} d G\left(\lambda_{t+1} ; \lambda_{t}\right)$ is itself declining as a function of $q$ for fixed $\lambda_{t}$. Using the notation of Lemma 3.1 make, for fixed $\lambda_{t}$, the following identifications:

$$
\begin{aligned}
p() & =u^{\prime}(), \quad h()=\beta f^{\prime}() \int u^{\prime}\left(c\left(f() \lambda_{t+1}, \lambda_{t+1}\right)\right) \lambda_{t+1} d G\left(\lambda_{t+1} ; \lambda_{t}\right), \\
c\left(\gamma, \lambda_{t}\right) & =a(\gamma), \quad s\left(\gamma, \lambda_{t}\right)=b(\gamma) .
\end{aligned}
$$


Clearly, $p(\gamma)>0, h(\gamma)>0, p^{\prime}(\gamma)<0, h^{\prime}(\gamma)<0$, and the various limit conditions are satisfied. Since $\gamma u^{\prime \prime}(\gamma) / u^{\prime}(\gamma)$ is either constant or increasing as $\gamma \mapsto \infty$, then $u^{\prime}(\gamma) / \gamma u^{\prime \prime}(\gamma)$ is either constant or decreasing as $\gamma \mapsto \infty$. Therefore, by our identification, $p(\gamma) / \gamma p^{\prime}(\gamma)$ is either constant or decreasing as $\gamma \mapsto \infty$. It remains to show that $h^{\prime}(\gamma)<p^{\prime}(\gamma)<0$ and that $\left|h^{\prime}(\gamma) / p^{\prime}(\gamma)\right|>N$ on $(\varepsilon, M)$. Represent $h(\gamma)$ as $h(\gamma)=f^{\prime}(\gamma) L(\gamma)$, where $L(\gamma)=\beta \int u^{\prime}\left(c\left(f(\gamma) \lambda_{t+1}, \lambda_{t+1}\right)\right) \lambda_{t+1} d G\left(\lambda_{t+1} ; \lambda_{t}\right)$. Now, for any $K>1$, we may, without loss of generality, assume $L(\gamma)>K, \forall \gamma$ in $(\varepsilon, M)$. This is due to the boundedness of $f()$ and thus also of $f() \bar{\lambda}$ which allows us to assert that $\forall t$, there exists a consumption level $\bar{c}$ such that $c_{t} \leqslant \bar{c}$. By scaling $u()$ so that $u^{\prime}(\bar{c}) \geqslant K$ (and in no way altering preferences) we can ensure $L(\gamma)>K$, $\forall \gamma$. As a result,

$$
h^{\prime}(\gamma)=f^{\prime \prime}(\gamma) L(\gamma)+f^{\prime}(\gamma) L^{\prime}(\gamma)<f^{\prime \prime}(\gamma)<u^{\prime \prime}(\gamma)=p^{\prime}(\gamma) .
$$

Furthermore, for any interval $(\tilde{\varepsilon}, M), \tilde{\varepsilon}>0$ and any $\gamma \in(\tilde{\varepsilon}, M)$,

$$
\left|\frac{h^{\prime}(\gamma)}{p^{\prime}(\gamma)}\right|=\left|\frac{f^{\prime \prime}(\gamma) L(\gamma)+f^{\prime}(\gamma) L^{\prime}(\gamma)}{p^{\prime}(\gamma)}\right|>\left|\frac{f^{\prime \prime}(\gamma) L(\gamma)}{p^{\prime}(\gamma)}\right|>K\left|\frac{f^{\prime \prime}(\gamma)}{p^{\prime}(\gamma)}\right| .
$$

Now on any interval $(\tilde{\varepsilon}, M)$, inf ${ }_{y}\left|f^{\prime \prime}(\gamma) / p^{\prime}(\gamma)\right|=P>1$. Without loss of generality, choose the scale factor $K$ so that $K P>N$. Thus, by an application of Lemma 3.1, we may assert $\left(c\left(q, \lambda_{t}\right) / q\right) \uparrow$ as $q \uparrow,\left(s\left(q, \lambda_{t}\right) / q\right) \downarrow$ as $q \uparrow$, for fixed $\lambda_{t}$, arbitrary, and $q \in(\varepsilon, M)$.

Remark. The assumptions of constant or declining risk aversion lower bounds $u^{\prime \prime \prime}(\cdot) /\left|u^{\prime \prime}(\cdot)\right|$. However, this is not inconsistent with our assumption in Theorem 3.2 since $K$ and hence $(N-1) / M$ can be made arbitrarily large. Taken together, Theorems 3.1 and 3.2 suggest that the savings functions assume the general forms of Fig. 3 .

Three comments are in order. First, the conclusions of Theorem 3.2 support what is observed-as nations become wealthier, they typically save (invest) a smaller fraction of their national output.

Secondly, given the complexity of the first order condition, it is indeed fortuitous that the restrictions on preferences (A.3) can be so conveniently expressed as restrictions on the agent's measure of relative risk aversion. Furthermore, the restriction that $u^{\prime \prime}(\cdot)>f^{\prime \prime}(\cdot)$, while clearly too strong (in simulation work with a large class of functional forms, the conclusions of Theorem 3.2 are observed without this restriction being satisfied), is nevertheless not unnatural. A chief distinction between stochastic growth models such as the one studied here, and their certainty analogue, is the influence of preferences in determining the steady state. It is then reasonable to expect that joint relationships between preferences and technology would influence the form of the savings function. 


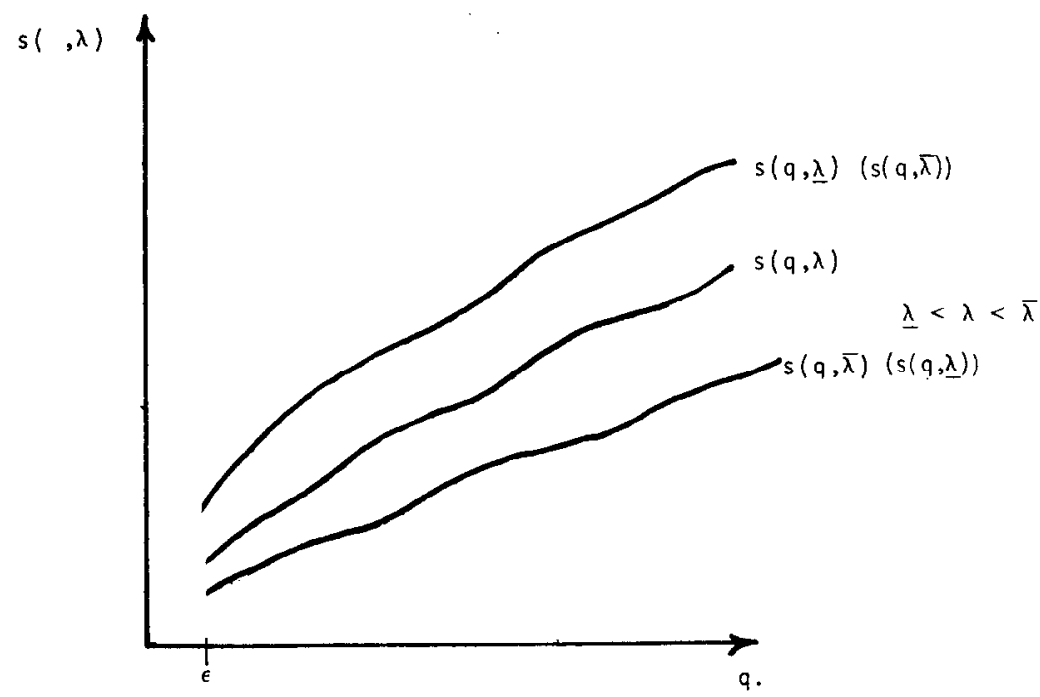

Figure 3

Lastly, we point out that the arguments of Lemma 3.1 and Theorem 3.2 could equally well be applied to settings for which $\lambda$ is i.i.d. through time (as is done in Danthine and Donaldson [9]). In particular, if the random shock is assumed to impact preferences (i.e., $u(\cdot)=u\left(c_{t}, \lambda_{t}\right), \lambda_{t}$ i.i.d.), the resultant dependence of the savings function on $\lambda_{t}, s=s\left(q_{t}, \lambda_{t}\right)$, also requires the additional structure provided by the conclusion to Theorem 3.2 in order to generate convergence (see, once again, Danthine and Donaldson [9]).

\section{Convergence}

We follow the approach of Mendelssohn and Sobel [21] as adapted from Feller $[12]$. In order to prove that the stochastic process on output (equivalently, capital stock) converges to a stationary distribution on some unique finite interval $[q, \bar{q}]$ (correspondingly, some interval $[\underline{k}, \bar{k}]$ ) it is sufficient to demonstrate that there exists only one "stable interval" $[\underline{q}, \bar{q}]$. This can be shown as follows.

(i) Equicontinuity: the family of measures $\left\{\mu_{t}()\right\}$ defined by $\mu_{t}\left(q^{\prime}\right)=$ $\int k\left(q, q^{\prime}\right) \mu_{t-1}(q), \mu_{0}$ given, is equicontinuous on $[\underline{q}, \bar{q}]$, where $k\left(q, q^{\prime}\right)$ is the density function of the stochastic kernel (to be defined) of the Markov process on output.

(ii) Ergodicity of $[q, \bar{q}]$ : intervals disjoint from $[\underline{q}, \bar{q}]$ are transient subsets of the process. 
(iii) Irreducibility: the process is irreducible (to be defined) on $[\underline{q}, \bar{q}]$.

It is easier to show that the Markov process on capital stock satisfies properties (ii) and (iii) and this is what we do. However, these properties then carry over, in a natural way, to the output process as well (see Section II for the capital stock/output correspondence).

We now turn to a consideration of these issues.

(i) Equicontinuity. The stochastic kernel $K\left(q, q^{\prime}\right)$ of the Markov process defined on aggregate output is defined by

$$
\begin{aligned}
K\left(q, q^{\prime}\right) & =\operatorname{Prob}\left(q_{t+1} \leqslant q^{\prime} \mid q_{t}=q\right) \\
& =\operatorname{Prob}\left(f\left(s\left(q, \lambda_{t}\right)\right) \lambda_{t+1} \leqslant q^{\prime}\right) \\
& =\operatorname{Prob}\left(s\left(q, \lambda_{t}\right) \leqslant f^{-1}\left(q^{\prime} / \lambda_{t+1}\right)\right) ;
\end{aligned}
$$

it must be shown, as a preliminary first step, to be continuous.

For a given $q$, aggregate savings $s\left(q, \lambda_{t}\right)$ is one to one, monotonically decreasing and continuously differentiable as a function of $\lambda_{t}$. Thus $s_{\lambda}^{-1}\left(q, \lambda_{t}\right)$, defined by the equation

$$
s\left(q, s_{\lambda}^{-1}\left(q, \lambda_{t}\right)(\theta)\right)=\theta,
$$

is continuously differentiable as a function of $\lambda_{t}$. The stochastic kernel $K\left(q, q^{\prime}\right)$ may be represented by

$$
K\left(q \cdot q^{\prime}\right)=\int_{\lambda_{t+1}=1}^{\lambda} \int_{\lambda_{t}=s_{\lambda}^{-1}\left(q, \lambda_{t}\right)\left(f^{-1}\left(q^{\prime} / \lambda_{t+1}\right)\right)}^{\lambda} d G\left(\lambda_{t+1} ; \lambda_{t}\right) .
$$

Thus,

$$
\begin{aligned}
k\left(q, q^{\prime}\right)= & \frac{\partial K\left(q, q^{\prime}\right)}{\partial q^{\prime}} \\
= & \frac{\partial}{\partial q^{\prime}}\left[\int_{\lambda_{t+1}=1}^{\lambda} \int_{\lambda_{t}=s_{\lambda}^{-1}\left(q, \lambda_{t}\right)\left(f^{-1}\left(q^{\prime} / \lambda_{t+3}\right)\right)}^{\lambda} d G\left(\lambda_{t+1} ; \lambda_{t}\right)\right] \\
= & \int_{\lambda_{t+1}=1}^{\lambda}-\left\{d G \left(\lambda_{t+1} ; s_{\lambda}^{-1}\left(q, \lambda_{t}\right)\left(f^{-1}\left(\frac{q^{\prime}}{\lambda_{t+1}}\right)\right)\right.\right. \\
& \left.\cdot \frac{d}{d q^{\prime}}\left[s_{\lambda}^{-1}\left(q, \lambda_{t}\right)\left(f^{-1}\left(\frac{q^{\prime}}{\lambda_{t+1}}\right)\right)\right]\right\} .
\end{aligned}
$$

By the inverse function theorem $d / d q^{\prime}\left(s_{\lambda}^{-1}\left(q, \lambda_{t}\right)\left(f^{-1}\left(q^{\prime} / \lambda_{t+1}\right)\right)\right)$ is continuous, while $-d G\left(\lambda_{t+1}, s_{\lambda}^{-1}\left(q, \lambda_{t}\right)\left(f^{-1}\left(q^{\prime} / \lambda_{t+1}\right)\right)\right.$ is continuous as it is the composition of continuous functions; the desired continuity follows. 
Given the continuity of this density function, the equicontinuity of the recursively defined family of measures $\left\{\mu_{t}()\right\}$,

$$
\mu_{t}\left(q^{\prime}\right)=\int k\left(q, q^{\prime}\right) \mu_{t-1}(q),
$$

with $\mu_{0}$ uniformly continuous, is easily proved on any closed interval $[\hat{q}, \hat{q}]$ :

Let $M=\max _{q \in[\hat{q}, \hat{q}]}\left|u_{0}(q)\right|$. By the recursive definition of $\mu_{t}(), \forall t$, $\left|\mu_{t}(q)\right| \leqslant M$. Then

$$
\begin{aligned}
\left|\mu_{t}(q)-\mu_{t}\left(q^{\prime}\right)\right| & =\left|\int k(v, q) \mu_{t-1}(v) d v-\int k\left(v, q^{\prime}\right) \mu_{t-1}(v) d v\right| \\
& \leqslant \int\left|k(v, q)-k\left(v, q^{\prime}\right)\right|\left|\mu_{t-1}(v)\right| d v \\
& <\varepsilon
\end{aligned}
$$

for some $\delta$ sufficiently small to ensure $\left|q-q^{\prime}\right|<\delta \Rightarrow\left|k(v, q)-k\left(v, q^{\prime}\right)\right|<$ $\varepsilon / M, \forall v$. Such a $\delta$ is possible as $k($,$) is uniformly continuous on [\hat{q}, \hat{q}]$.

(ii) Ergodicity of $[k, \bar{k}]$ : equivalently of $[q, \bar{q}]$. As mentioned earlier, we will alternatively work with the capital stock and output series.

Focusing on the output series, we first note that the range of the stationary distribution on output has, thus far, not been defined. Our initial task, then, is to show this range is bounded above by some $\bar{q}<\infty$ (equivalently $\bar{k}<\infty$ ), and that it is not degenerate, that is $\bar{q}>0$ (equivalently $\bar{k}>0$ ), an issue considered in the following lemma:

LEMMA 4.1. Let $\bar{q}$ be the upper bound on the stationary distribution on output. Then $0<\bar{q}<\infty$.

Proof. That $\bar{q}<\infty$ comes directly from the boundedness of the production technology. To show that $\bar{q}>0$, assume rather that for some $\varepsilon>0, f(s(q, \lambda)) \lambda^{\prime}<q \forall \lambda, \lambda^{\prime}, \forall q \in[0, \varepsilon]$. Suppose that at time $t, q_{t}<\varepsilon$, and consider some arbitrary evolution of output $\left\{q_{t+n}\right\}, n=1,2,3, \ldots$, corresponding to some sequence of random shocks $\left\{\lambda_{t+n}\right\}$. By the optimality condition, $\forall n$,

$$
\begin{aligned}
u^{\prime}\left(c\left(q_{t+n}, \lambda_{t+n}\right)\right)= & \beta f^{\prime}\left(s\left(q_{t+n}, \lambda_{t+n}\right)\right) \int u^{\prime}\left(c \left(f\left(s\left(q_{t+n}, \lambda_{t+n}\right)\right)\right.\right. \\
& \left.\left.\cdot \lambda_{t+n+1}, \lambda_{t+n+1}\right)\right) \lambda_{t+n+1} d G\left(\lambda_{t+n+1} ; \lambda_{t+n}\right) .
\end{aligned}
$$

Since $\forall \lambda_{t+n+1}, f\left(s\left(q_{t+n}, \lambda_{t+n}\right)\right) \lambda_{t+n+1}<q_{t+n}$, we claim $\exists$ a subsequence $n_{k}$, $k=1,2, \ldots$, for which

$$
\begin{aligned}
u^{\prime}\left(c\left(q_{t+n_{k}}, \lambda_{t+n_{k}}\right)\right)< & \int u^{\prime}\left(c\left(f\left(s\left(q_{t+n_{k}}, \lambda_{t+n_{k}}\right)\right) \lambda_{t+n_{k}+1}, \lambda_{t+n_{k}+1}\right)\right) \\
& \cdot \lambda_{t+n_{k}+1} d G\left(\lambda_{t+n_{k}+1} ; \lambda_{t+n_{k}}\right) .
\end{aligned}
$$


To show that such a subsequence must exist with probability one, suppose, to the contrary that $\forall j>t$,

(i) $u^{\prime}\left(c\left(q_{j}, \lambda_{j}\right)\right) \geqslant \int u^{\prime}\left(c\left(f\left(s\left(q_{j}, \lambda_{j}\right)\right) \lambda_{j+1}, \lambda_{j+1}\right)\right) \lambda_{j+1} d G\left(\lambda_{j+1} ; \lambda_{j}\right)$,

where by assumption,

(ii) $f\left(s\left(q_{j}, \lambda_{j}\right)\right) \lambda_{j+1}<q_{j}, \forall \lambda_{j+1}$.

Furthermore, we may assume that $\underline{\lambda}$ is as large as we like, so that the $\lambda_{j+1}$ coefficients of the $u^{\prime}()$ terms on the right-hand side of (i) are as large as we like. To see this, note that our production technology $f() \lambda$ may be rewritten, for arbitrary constant $\omega>0$ as $((1 / \omega) f()) \omega \lambda$.

Such a procedure would leave the optimal consumption and savings policy functions unchanged so that $\forall \omega, \forall q \quad c(q, \lambda)=c^{\omega}(q, \omega \lambda)$, where the superscript $\omega$ indicates the solution to $P$ with technology $((1 / \omega) f()) \omega \lambda$.

If $\underline{\lambda}$ is chosen sufficiently large, then for a given small $\varepsilon>0$, such that $\lambda+\varepsilon<\bar{\lambda}$, inequality (i) can hold if $\forall j>t$,

$$
\lambda_{j} \in[\underline{\lambda}, \underline{\lambda}+\varepsilon) .
$$

However, this occurs with probability zero. By the optimality condition, Eq. (2) thus implies that

$$
1>\beta f^{\prime}\left(s\left(q_{t+n_{k}}, \lambda_{t+n_{k}}\right)\right), \quad k=1,2,3, \ldots
$$

But this inequality would be violated if $q_{t+n_{k}} \rightarrow 0$ as $n_{k} \rightarrow \infty$ since this implies $s\left(q_{t+n_{k}}, \lambda_{t+n_{k}}\right) \rightarrow 0$ as $n_{k} \rightarrow \infty$.

It would be convenient to be able to assert that $q>0(k>0)$, but this is not possible given our current structure. In order to secure a positive lower bound on output in the i.i.d. case, Brock and Mirman [5] require that positive probability be assigned to the lower bound on the (i.i.d.) random shocks. The analogous condition in our model would be to require $d G(\lambda, \lambda)>0$, a condition which would somewhat complicate our analysis.

This issue of the positivity of the lower bound is exhaustively considered in Mirman and Zilcha [24], where they show that it is generally possible for the stochastic process generated by an optimal policy to have support on an interval with zero as an end point. However, as they also point out, (Mirman and Zilcha [24, p. 127]), the difficulty in securing an explicit example in which this is observed suggests that the phenomenon is largely pathological. ${ }^{5}$

${ }^{5}$ There is a sufficient condition in Brock $|4|$ to exclude this problem in Mirman and Zilcha $|24|$. It may be possible to extend this condition to the case of serially correlated disturbances. 
In this light, we will assume $q>0$ (our argument may be adapted, however, to admit $\underline{q}=0$ ); equivalently $\underline{k}>0$.

It remains to identify $q$ and $\bar{q}$ (equivalently $\underline{k}$ and $\bar{k}$ ) and to ensure that the ergodic set is, in fact, a closed interval. An analysis of these questions reduces to an examination of the capital stock transition function $s\left(f\left(k_{t}\right) \lambda_{t}, \lambda_{t}\right)$, where $k_{t+1}=s\left(f\left(k_{t}\right) \lambda_{t}, \lambda_{t}\right)$. To identify, as our first task, $\bar{k}$ and $\underline{k}$, we need only eliminate the possibility that these transition functions cross for distinct values of $\lambda$, where, say, $\lambda^{\prime}>\lambda^{\prime \prime}>\lambda^{\prime \prime \prime}$ (see Fig. 4). Such a possibility cannot be a priori ruled out because of the properties of the savings function $s\left(q_{t}, \lambda_{t}\right)$. Although $s(\cdot, \cdot)$ is an increasing function of $q_{t}$ for fixed $\lambda_{t}$, it may be decreasing as a function of $\lambda_{t}$ for fixed $q_{t}$ (see Theorem 3.1) and it is not clear which effect dominates. Lemma 4.2 shows that the former effect in all cases dominates the latter; it forms the basis of Theorem 4.1 to follow.

LeMmA 4.2. Under assumptions A.1-A.4, for any $\lambda_{2}, \lambda_{1}$, and $k$, such that $\lambda_{2}>\lambda_{1}$ we have $s\left(f(k) \lambda_{2}, \lambda_{2}\right)>s\left(f(k), \lambda_{1}, \lambda_{1}\right)$, provided $L$ is sufficiently small (see A.1 for the definition of $L$ ).

Proof. Since, by assumption, $q>0$ and since consumption is an increasing function of output, there is a $\subseteq$ such that $c_{t} \geqslant c \forall t$. Hence by A.3, $\exists$ an $M$ such that $-u^{\prime \prime}\left(c_{t}\right)<M \forall c_{\text {, }}$.

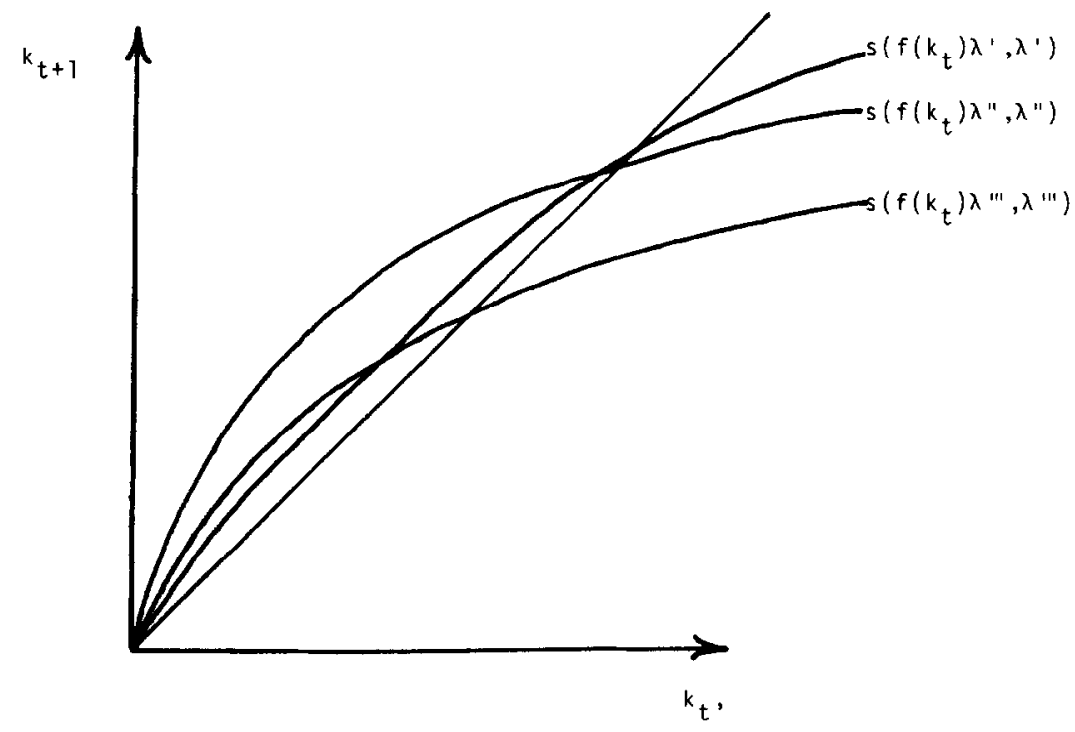

Figure 4 
Given a $\lambda_{1}, c\left(d(k), \lambda_{1}, \lambda_{1}\right)$ and $s\left(f(k) \lambda_{1}, \lambda_{1}\right)$ are defined by:

$$
\begin{aligned}
& u^{\prime}\left(c\left(f(k) \lambda_{1}, \lambda_{1}\right)\right) \\
& \quad=\beta f^{\prime}\left(s\left(f(k) \lambda_{1}, \lambda_{1}\right)\right) \int u^{\prime}\left(c\left(f\left(s\left(f(k) \lambda_{1}, \lambda_{1}\right)\right) \lambda, \lambda\right)\right) \lambda d G\left(\lambda ; \lambda_{1}\right) .
\end{aligned}
$$

Consider a shift from $\lambda_{1}$ to $\lambda_{2}$; if the conclusion to the lemma were not to hold, for this $\lambda_{2}$, then

$$
s\left(f(k) \lambda_{2}, \lambda_{2}\right) \leqslant s\left(f(k), \lambda_{1}, \lambda_{1}\right),
$$

and

$$
c\left(f(k) \lambda_{2}, \lambda_{2}\right) \geqslant c\left(f(k) \lambda_{1}, \lambda_{1}\right)+f(k)\left(\lambda_{2}-\lambda_{1}\right) .
$$

By the assumption on $u^{\prime \prime}()$,

$$
u^{\prime}\left(c\left(f(k) \lambda_{1}, \lambda_{1}\right)\right)-u^{\prime}\left(c\left(f(k) \lambda_{2}, \lambda_{2}\right)\right) \geqslant M f(k)\left(\lambda_{2}-\lambda_{1}\right),
$$

or

$$
u^{\prime}\left(c\left(f(k) \lambda_{2}, \lambda_{2}\right)\right)+M f(k)\left(\lambda_{2}-\lambda_{1}\right) \leqslant u^{\prime}\left(c\left(f(k) \lambda_{1}, \lambda_{1}\right)\right) .
$$

Furthermore,

$$
\beta f^{\prime}\left(s\left(f(k) \lambda_{2}, \lambda_{2}\right)\right) \geqslant \beta f^{\prime}\left(s\left(f(k) \lambda_{1}, \lambda_{1}\right)\right)
$$

and

$$
\beta f^{\prime}\left(s\left(f(k) \lambda_{2}, \lambda_{2}\right)\right) \leqslant \beta \max \left[f^{\prime}\left(k_{0}\right), f^{\prime}\left(\frac{k}{2}\right)\right] \equiv M_{1}
$$

( $k_{0}$ is the initial capital).

and $\forall \lambda_{t}, \lambda_{t+1}$, we may assume that

$$
u^{\prime}\left(c\left(f\left(s\left(f(k) \lambda_{t}, \lambda_{t}\right)\right) \lambda_{t+1}, \lambda_{t+1}\right)\right) \leqslant u^{\prime}\left(\frac{c}{2}\right) \equiv M_{2} .
$$

Lastly,

$$
\begin{aligned}
& \beta f^{\prime}\left(s\left(f(k) \lambda_{1}, \lambda_{1}\right)\right) \int u^{\prime}\left(c\left(f\left(s\left(f(k) \lambda_{1}, \lambda_{1}\right)\right) \lambda, \lambda\right)\right) \lambda d G\left(\lambda ; \lambda_{1}\right) \\
& \quad-\beta f^{\prime}\left(s\left(f(k) \lambda_{2}, \lambda_{2}\right)\right) \int u^{\prime}\left(c\left(f\left(s\left(f(k) \lambda_{2}, \lambda_{2}\right)\right) \lambda, \lambda\right)\right) \lambda d G\left(\lambda ; \lambda_{2}\right) \\
& <\beta f^{\prime}\left(s\left(f(k) \lambda_{1}, \lambda_{1}\right)\right) \int u^{\prime}\left(c\left(f\left(s\left(f(k) \lambda_{1}, \lambda_{1}\right)\right) \lambda_{1}, \lambda\right)\right) \lambda d G\left(\lambda ; \lambda_{1}\right) \\
& -\beta f^{\prime}\left(s\left(f(k) \lambda_{1}, \lambda_{1}\right)\right) \int u^{\prime}\left(c\left(f\left(s\left(f(k) \lambda_{1}, \lambda_{1}\right)\right) \lambda, \lambda\right)\right) \lambda d G\left(\lambda ; \lambda_{2}\right)
\end{aligned}
$$




$$
\begin{aligned}
= & \beta f^{\prime}\left(s\left(f(k) \lambda_{1}, \lambda_{1}\right)\right) \int u^{\prime}\left(c ( f ( s ( f ( k ) \lambda _ { 1 } , \lambda _ { 1 } ) \lambda , \lambda ) ) \lambda \left\{d G\left(\lambda ; \lambda_{1}\right)\right.\right. \\
& \left.-d G\left(\lambda ; \lambda_{2}\right)\right\} \\
\leqslant & \beta M_{1} M_{2} \bar{\lambda} \int\left|d G\left(\lambda, \lambda_{1}\right)-d G\left(\lambda, \lambda_{2}\right)\right| .
\end{aligned}
$$

Thus,

$$
\begin{aligned}
& u^{\prime}\left(c\left(f(k) \lambda_{2}, \lambda_{2}\right)\right)+M f(k)\left(\lambda_{2}-\lambda_{1}\right) \\
& \quad \leqslant u^{\prime}\left(c\left(f(k) \lambda_{1}, \lambda_{1}\right)\right) \\
& =\beta f^{\prime}\left(s\left(f(k) \lambda_{1}, \lambda_{1}\right)\right) \int u^{\prime}\left(c\left(f\left(s\left(f(k) \lambda_{1}, \lambda_{1}\right)\right) \lambda, \lambda\right)\right) \lambda d G\left(\lambda ; \lambda_{1}\right) \\
& \quad \leqslant \beta f^{\prime}\left(s\left(f(k) \lambda_{2}, \lambda_{2}\right)\right) \int u^{\prime}\left(c\left(f\left(s\left(f(k) \lambda_{2}, \lambda_{2}\right)\right) \lambda, \lambda\right)\right) \lambda d G\left(\lambda ; \lambda_{2}\right) \\
& \quad+\beta M_{1} M_{2} \bar{\lambda} \int\left|d G\left(\lambda ; \lambda_{1}\right)-d G\left(\lambda ; \lambda_{2}\right)\right|
\end{aligned}
$$

To arrive at a contradiction, it is sufficient that

$$
M f(k)\left(\lambda_{2}-\lambda_{1}\right)>\beta M_{1} M_{2} \bar{\lambda} \int\left|d G\left(\lambda ; \lambda_{1}\right)-d G\left(\lambda ; \lambda_{2}\right)\right|
$$

or

$$
\lambda_{2}-\lambda_{1}>\frac{\beta M_{1} M_{2} \bar{\lambda}}{f(k) M} \int\left|d G\left(\lambda ; \lambda_{1}\right)-d G\left(\lambda ; \lambda_{2}\right)\right|
$$

or that

$$
\lambda_{2}-\lambda_{1}>\frac{\beta M_{1} M_{2} \bar{\lambda}}{(\underline{q} / 2) M} \int\left|d G\left(\lambda ; \lambda_{1}\right)-d G\left(\lambda ; \lambda_{2}\right)\right| .
$$

Choose $L<q / 2 M / \beta M_{1} M_{2} \bar{\lambda}$; contradiction. Therefore, (3) cannot hold and the lemma is proved. ${ }^{6}$

Lemma 4.2 thus allows us to identify $\bar{k}$ with $\bar{\lambda}$ and $\underline{k}$ with $\underline{\lambda}: \underline{k}=$ $s(f(\underline{k}) \underline{\lambda}, \underline{\lambda})$ and $\bar{k}=s(f(\bar{k}) \bar{\lambda}, \bar{\lambda})$.

${ }^{6}$ Notice that $M_{1}, M_{2}, \beta, q / 2$, and $M$ are all independent of the shock transition structure as they are either independent parameters or are determined by the analogous certainty problem with $\lambda_{t} \equiv \lambda$. 


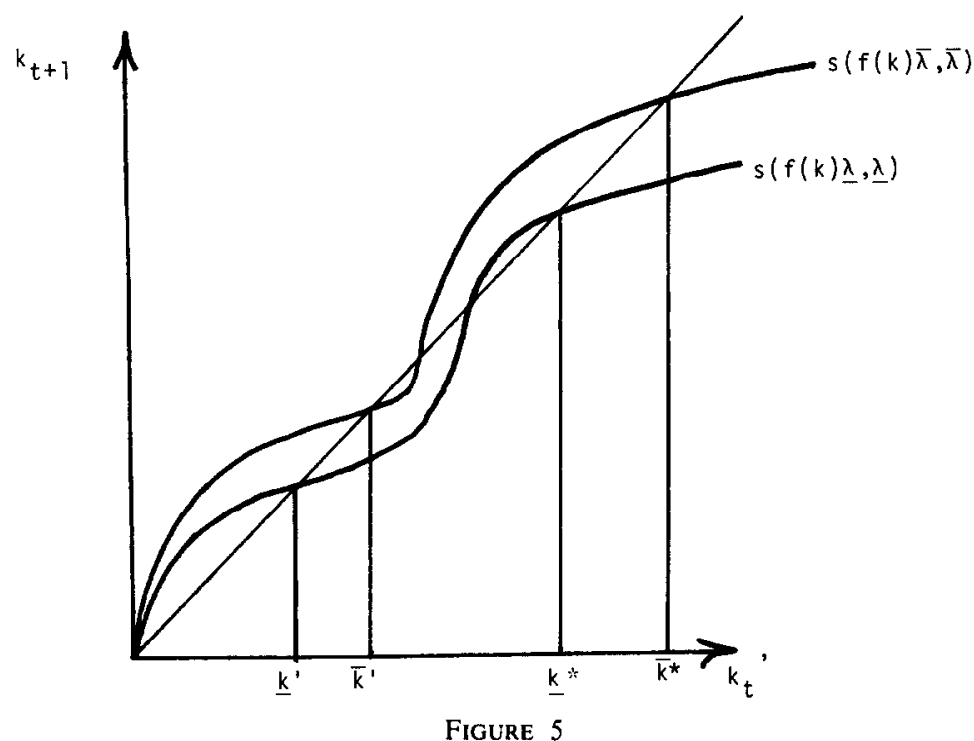

It remains to show that $k$ and $\vec{k}$ identify an ergodic set which is a unique interval. For example, if the transition function were to have the shape in Fig. 5. the process would evolve into one of two distinct ergodic sets $\left(\left[\underline{k}^{\prime}, \bar{k}^{\prime}\right]\right.$ or $\left[\underline{k}^{*}, \bar{k}^{*}\right]$ (see Fig. 5). Rather, conditions must be imposed to ensure that this family of functions conforms to one of the forms in Figs. 6 and 7 . In each of these cases the ergodic set will be a unique interval.

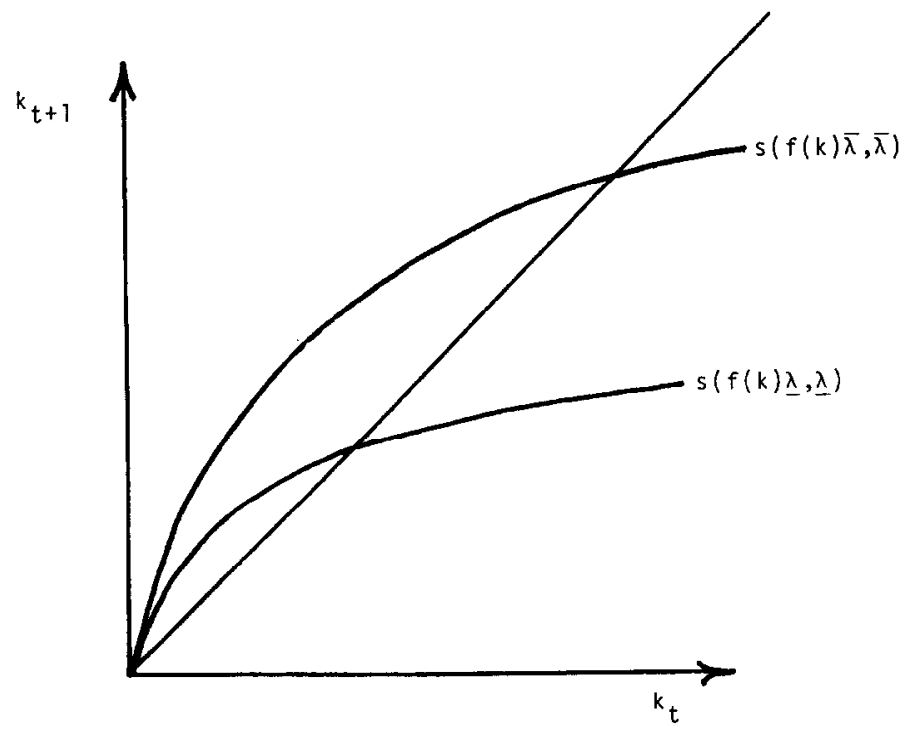

FiguRE 6 


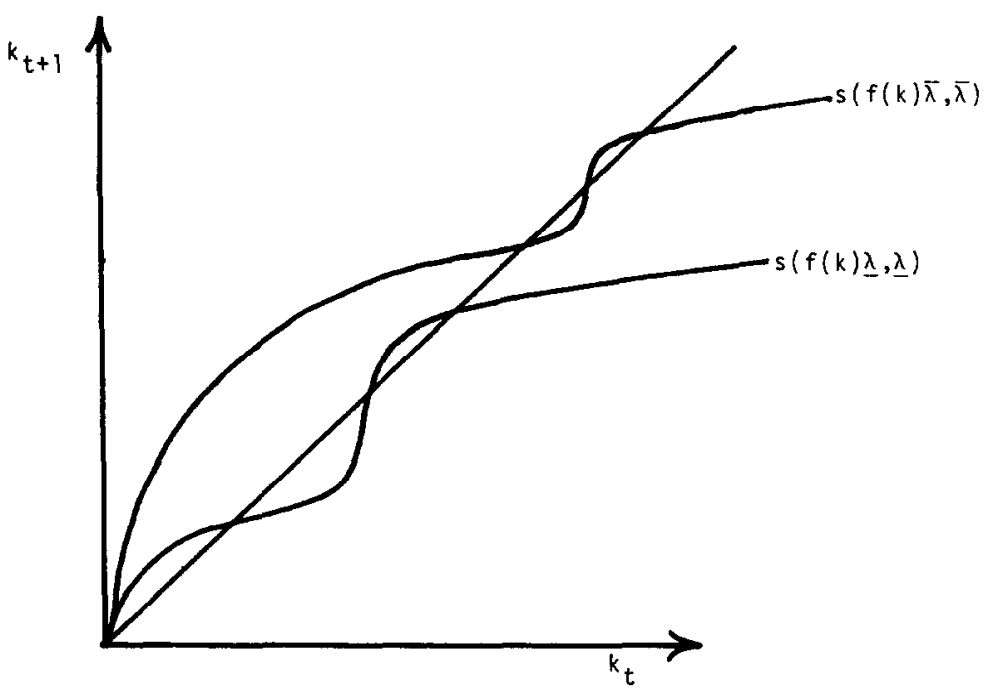

FIGURE 7

At this juncture the results of Theorem 3.2 become useful. Knowing that $s(q, \lambda) / q \backslash$ as $q \uparrow$ will enable us to show that the transition functions must essentially conform to the pattern suggested by Fig. 6-that for each $\lambda$, $s(f(k) \lambda, \lambda)$ crosses the $45^{\circ}$ line only once. Strictly speaking, however, Theorem 3.2 is not necessary-patterns (5) and (7) above can be eliminated in a manner identical to that given in Danthine and Donaldson (1981i).

LEMMA 4.3. Under the assumptions of Theorem 3.2, for each $\lambda$, there exists exactly one $k=k_{\lambda}$ at which $s\left(f\left(k_{\lambda}\right), \lambda\right)=k_{\lambda}$.

Proof. Since, for any $\lambda, f(k) \lambda$ is concave and bounded as a function of $k, f(k) \lambda / k \downarrow$ as $k \uparrow$ and thus $f^{\prime}(k) \lambda<f(k) \lambda / k$. Similarly since $s(q, \lambda) / q \downarrow$ as $q \uparrow, s^{\prime}(q, \lambda) q<s(q, \lambda)$. Letting $q=f(k) \lambda$ in the preceding inequality, yields

$$
s^{\prime}(f(k) \lambda, \lambda) f(k) \lambda<s(f(k) \lambda, \lambda)
$$

and thus

$$
s^{\prime}(f(k) \lambda, \lambda) f(k) \lambda\left[f^{\prime}(k) \lambda\right]<s(f(k) \lambda, \lambda)\left[\frac{f(k) \lambda}{k}\right]
$$

or

$$
s^{\prime}(f(k) \lambda, \lambda) f^{\prime}(k) \lambda k<s(f(k) \lambda, \lambda),
$$

or

$$
\frac{s^{\prime}(f(k) \lambda, \lambda) f^{\prime}(k) \lambda \cdot k-s(f(k) \lambda, \lambda)}{k^{2}}<0,
$$


or

$$
\frac{\partial}{\partial k}\left[\frac{s(f(k) \lambda, \lambda)}{k}\right]<0
$$

Thus, $\forall k \geqslant 0, s(f(k) \lambda, \lambda) / k \downarrow$ as $k \uparrow \forall \lambda$, and

$$
s^{\prime}(f(k) \lambda, \lambda) f^{\prime}(k) \lambda<\frac{s(f(k) \lambda, \lambda)}{k} .
$$

For a given $\hat{\lambda}$, let $k_{\lambda}$ be the smallest $k$ such that

$$
s\left(f\left(k_{\lambda}\right) \hat{\lambda}, \hat{\lambda}\right)=k_{\hat{\lambda}}
$$

or, equivalently, for which

$$
\frac{s\left(f\left(k_{\lambda}\right) \hat{\lambda}, \hat{\lambda}\right)}{k_{\hat{\lambda}}}=1 .
$$

But, since

$$
s^{\prime}\left(f\left(k_{\lambda}\right) \hat{\lambda}, \hat{\lambda}\right) f^{\prime}\left(k_{\lambda}\right) \hat{\lambda}<\frac{s\left(f\left(k_{\lambda}\right) \hat{\lambda}, \hat{\lambda}\right)}{k_{\lambda}}=1
$$

and since

$$
\begin{gathered}
\frac{s(f(k) \lambda, \lambda)}{k} \downarrow \text { as } k \uparrow, \quad \text { then } \forall k \geqslant k_{\lambda} \\
\frac{\partial}{\partial k}[s(f(k) \hat{\lambda}, \hat{\lambda})]<1 .
\end{gathered}
$$

But this ensures that once $s(f(k) \lambda, \lambda)$ crosses the $45^{\circ}$ line, which has slope one, it can never cross it again and the Lemma is proved.

Assured that the transition function satisfies the pattern suggested by Fig. 6, it is routine to demonstrate that the ergodic set of the Markov process on capital stock is the closed interval $[\underline{k}, \bar{k}]$, where $\underline{k}=s(f(\underline{k}) \underline{\lambda}, \underline{\lambda})$ and $\bar{k}=$ $s(f(\bar{k}) \bar{\lambda}, \bar{\lambda})$

THEOREM 4.1. If $\left[k_{1}, k_{2}\right] \cap[\underline{k}, \bar{k}]$ is empty then $\left[k_{1}, k_{2}\right]$ is a transient subset of the Markov process on output.

Proof. Suppose that $\left[k_{1}, k_{2}\right]$ lies to the right of $[\underline{k}, \bar{k}]$ and that, for some time $t, k_{t}=k_{0} \in\left[k_{1}, k_{2}\right]$; we must show that the process passes out of 
$\left[k_{1}, k_{2}\right]$. Consider any sequence $\left\{\lambda_{t+n}\right\}, n=1,2, \ldots$ and form the sequence $k_{t+n}$ and $\hat{k}_{t+n}$ defined, respectively, by

$$
\begin{gathered}
k_{t+n}=s\left(f\left(k_{t+n-1}\right) \lambda_{t+n-1}, \lambda_{t+n-1}\right), \\
\hat{k}_{t+n}=s\left(f\left(\hat{k}_{t+n-1}\right) \bar{\lambda}, \bar{\lambda}\right), \quad k_{t}=\hat{k}_{t}=k_{0} .
\end{gathered}
$$

By Lemma 4.3, $\forall n, k_{t+n}<\hat{k}_{t+n}$. By construction, however, $\hat{k}_{t+n} \rightarrow \bar{k}$, so that for some $N, k_{t+n}<k_{1}$ with probability 1 . Thus $\left[k_{1}, k_{2}\right]$ is a transient set of the process. A similar argument can be made for intervals $\left[k_{1}, k_{2}\right]$ to the left of $[\underline{k}, \bar{k}]$.

By direct analogy, the ergodic set of the Markov process on output is given by $[\underline{q}, \bar{q}]$ where $q=f(\underline{k}) \underline{\lambda}$ and $\bar{q}=f(\bar{k}) \bar{\lambda}$.

(iii) Irreducibility. We have to show that any open set $A \subseteq[\underline{k}, \bar{k}]$ can be reached from any point $k_{0} \in[\underline{k}, \bar{k}]$ in a finite number of steps with positive probability. This makes explicit the notion of irreducibility.

THEOREM 4.2. The Markov process on capital stock is irreducible on $[\underline{k}, \bar{k}]$.

Proof. Pick an open subset $A=\left(k_{1}, k_{2}\right) \subseteq[\underline{k}, \bar{k}]$, and a point $k_{0} \in[\underline{k}, \bar{k}]$, $k_{0} \notin A$. Since $A$ is open, for some $\hat{k} \in A$, we can find a neighborhood $J(\hat{k}, \varepsilon)=(\hat{k}-\varepsilon, \hat{k}+\varepsilon)$ such that $J(\hat{k}, \varepsilon) \subseteq A$ and thus $\hat{k}-\varepsilon>k_{1}$. Consider the following set

$$
J^{\lambda}(\hat{k}, \varepsilon)=\{\lambda: s(f(k) \lambda, \lambda)=k \quad \text { for some } \quad k \in J(\hat{k}, \varepsilon)\} .
$$

By our assumptions on the transition function for $\lambda$, for any $\lambda^{0}$,

$$
\int_{\lambda_{\lambda(\mathbb{k}, \varepsilon)}} d G\left(\lambda ; \lambda^{0}\right)=\mu>0
$$

and

$$
\begin{aligned}
\operatorname{Prob}\left(\lambda_{t+1} \in J^{\lambda}(\hat{k}, \varepsilon) \mid \lambda_{t} \in J^{\lambda}(\hat{k}, \varepsilon)\right) & =\int_{\lambda_{t+1} \in J \lambda\left(k_{,} \varepsilon\right)} \int_{\lambda_{t} \in J \lambda(k, \varepsilon)} d G\left(\lambda_{t+1} ; \lambda_{t}\right) \\
& =\hat{\mu}>0 .
\end{aligned}
$$

Suppose that at some time $t, k_{t}=k_{0}$, and for each $\lambda \in J^{\lambda}(\hat{k}, \varepsilon)$, consider the sequence $\left\{k_{t+n}^{\lambda}\right\}, n=1,2,3, \ldots$ defined by $k_{t+n}^{\lambda}=s\left(f\left(k_{t+n-1}^{\lambda}\right) \lambda, \lambda\right) ; k_{t+1}^{\lambda}=$ $s\left(f\left(k_{0}\right) \lambda, \lambda\right)$. For each $\lambda$, there is a $T(\lambda)$ such that for $n \geqslant T(\lambda), k_{t+n}^{\lambda} \geqslant k_{1}$, and let $T^{*}=\sup _{\lambda \in J \lambda\left(k_{\varepsilon}\right)}\{T(\lambda)\}$. By construction, $T^{*}<\infty$. Thus, $P\left(k_{t+T^{*}} \in A \mid k_{t}=k_{0}\right) \geqslant \hat{\mu}^{T^{*}} \cdot \mu>0$. 
Once again, by direct analogy we may also assert that the Markov process on output is irreducible on $[q, \bar{q}]$ as defined earlier. Thus, the economy will evolve to the same asymptotic stationary state (distribution) on output irrespective of the initial output value; similarly for capital stock and consumption. Our convergence argument does not, in its overall thrust, differ substantially from that of the i.i.d. case. As mentioned earlier, the restrictions on relative risk aversion and the requirement that $f^{\prime \prime}(\gamma)\left\langle u^{\prime \prime}(\gamma) \forall \gamma>0\right.$ are unnecessary (as the results of Theorem 3.2 are unnecessary for convergence of capital stock). They were included for other purposes. Thus, A.1 is the only assumption which of necessity differs substantially from the i.i.d. case.

\section{EfFects of Changes in Information on Consumption/Savings Policies}

This section could equally well be entitled "effects of changes in expectations on consumption/savings policies." It considers the issue of how changes in the conditional distribution function $G\left(\lambda_{t+1} ; \lambda_{t}\right)$ affect the stationary consumption/savings policies. Two representative changes are considered. In the first we examine the effects of "greater persistence" such as would be suggested by, in the discrete case, a shift in the transition matrix as indicated below:

$$
\begin{aligned}
& \begin{array}{llll}
\lambda & \bar{\lambda} & \lambda & \bar{\lambda}
\end{array} \\
& \frac{\lambda}{\bar{\lambda}}\left[\begin{array}{ll}
.6 & .4 \\
.4 & .6
\end{array}\right] \rightarrow \frac{\lambda}{\lambda}\left[\begin{array}{ll}
.8 & .2 \\
.2 & .8
\end{array}\right] \text {. }
\end{aligned}
$$

The second considers the effects of uniformly more favorable expectations; that is, a shift in $G\left(\lambda_{t+1} ; \lambda_{t}\right)$ from $G^{1}\left(\lambda_{t+1} ; \lambda_{t}\right)$ to $G^{2}\left(\lambda_{t+1} ; \lambda_{t}\right)$, where $G^{2}\left(\lambda_{t+1} ; \lambda_{t}\right)$ stochastically dominates $G^{1}\left(\lambda_{t+1} ; \lambda_{t}\right)$ for all $\lambda_{t} \in[\underline{\lambda}, \bar{\lambda}]$.

These considerations are meant to be illustrative of one of the principal contributions of this study: If a certain qualitative change in savings behavior is desired, one may seek those changes in expectations (as summarized by the stochastic kernel or transition matrix of the random shock) necessary to induce such an effect. In this way one could, for example, construct a simple theory to describe the effects of changes in government policies, via their corresponding expectational effects, on aggregate private consumption/savings behavior. Rather than precisely formalizing our results, we limit ourselves to a discussion of our conclusions and the intuition behind them.

First consider the issue of greater persistence. It is difficult to describe such a shift in a simple way. For the discrete case, one could formalize a 
shift from $G^{1}\left(\lambda_{t+1} ; \lambda_{t}\right)$ to $G^{2}\left(\lambda_{t+1} ; \lambda_{t}\right)$, where $G^{2}\left(\lambda_{t+1} ; \lambda_{t}\right)$ illustrates the greater persistence by requiring $\forall \hat{\lambda}_{t} \in[\underline{\lambda}, \bar{\lambda}]$,

$$
\begin{aligned}
& P_{r}^{1}\left(\lambda_{t+1}=\hat{\lambda}_{t} ; \lambda_{t}=\hat{\lambda}_{t}\right)<P_{r}^{2}\left(\lambda_{t+1}=\hat{\lambda}_{t} ; \lambda_{t}=\hat{\lambda}_{t}\right), \\
& \quad P_{r}^{1}\left(\lambda_{t+1}=\lambda ; \lambda_{t}=\hat{\lambda}_{t}\right)>P_{r}^{2}\left(\lambda_{t+1}=\lambda ; \lambda_{t}=\hat{\lambda}_{t}\right) ; \quad \forall \lambda_{t} \neq \hat{\lambda} .
\end{aligned}
$$

For a continuous distribution, one could analogously require that for each $\hat{\lambda} \in[\underline{\lambda}, \bar{\lambda}]$, there exists a neighborhood $(\hat{\lambda}-\varepsilon, \hat{\lambda}+\varepsilon) \subseteq[\underline{\lambda}, \bar{\lambda}]$ (or, if $\hat{\lambda}=\underline{\lambda}$, a region $[\underline{\lambda}, \bar{\lambda}+\varepsilon)$; if $\lambda=\bar{\lambda}$, a region $(\bar{\lambda}-\varepsilon, \bar{\lambda}])$ such that $\forall \lambda_{t+1} \in(\hat{\lambda}-\varepsilon$, $\hat{\lambda}+\varepsilon)$.

$$
\begin{aligned}
& d G^{2}\left(\lambda_{t+1} ; \hat{\lambda}_{t}\right)>d G^{1}\left(\lambda_{t+1} ; \hat{\lambda}_{t}\right), \\
& d G^{2}\left(\lambda_{t+1} ; \hat{\lambda}_{t}\right)<d G^{1}\left(\lambda_{t+1} ; \hat{\lambda}_{t}\right), \quad \lambda_{t+1} \notin(\hat{\lambda}-\varepsilon, \hat{\lambda}+\varepsilon) .
\end{aligned}
$$

To get a feeling for the effects of such changes on the optimal policies, it is instructive to examine the effects at the boundaries $\underline{\lambda}$ and $\bar{\lambda}$. Let $s_{1}(q, \lambda)$ and $s_{2}(q, \lambda)\left(c_{1}(q, \lambda) ; c_{2}(q, \lambda)\right)$ represent the optimal savings (consumption) policies associated with, respectively, $G^{1}\left(\lambda_{t+1} ; \lambda_{t}\right)$ and $G^{2}\left(\lambda_{t+1} ; \lambda_{t}\right)$ as described above. Then $s_{i}(q, \bar{\lambda})$ and $c_{i}(q, \bar{\lambda})$ satisfy:

$$
\begin{aligned}
u^{\prime}\left(c_{t}\left(q_{t}, \lambda_{t}\right)\right)= & \beta f^{\prime}\left(s_{i}\left(q_{t}, \lambda_{t}\right)\right) \int u^{\prime}\left(c \left(f\left(s\left(q_{1}, \lambda_{t}\right)\right)\right.\right. \\
& \left.\left.\times \lambda_{t+1}, \lambda_{t+1}\right)\right) \lambda_{t+1} G^{1}\left(\lambda_{t+1} ; \lambda_{t}\right) . \text { For } i=1,2 .
\end{aligned}
$$

Suppose $\lambda_{t}=\bar{\lambda} ;$ then

$$
\begin{aligned}
& \int u^{\prime}\left(c_{1}\left(f\left(s_{1}\left(q_{t}, \bar{\lambda}\right)\right) \lambda_{t+1}, \lambda_{t+1}\right)\right) \lambda_{t+1} d G^{1}\left(\lambda_{t+1} ; \bar{\lambda}\right) \\
& \quad>\int u^{\prime}\left(c_{1}\left(f\left(s_{1}\left(q_{t}, \bar{\lambda}\right)\right) \lambda_{t+1}, \lambda_{t+1}\right)\right) \lambda_{t+1} d G^{2}\left(\lambda_{t+1} ; \bar{\lambda}\right)
\end{aligned}
$$

so that

$$
\begin{aligned}
u^{\prime}\left(c_{1}\left(q_{t}, \bar{\lambda}\right)\right)> & \beta f^{\prime}\left(s_{1}\left(q_{t}, \bar{\lambda}\right)\right) \int u^{\prime}\left(c _ { 1 } \left(f\left(s_{1}\left(q_{t}, \bar{\lambda}\right)\right)\right.\right. \\
& \left.\left.\times \lambda_{t+1}, \lambda_{t+1}\right)\right) \lambda_{t+1} d G^{2}\left(\lambda_{t+1} ; \bar{\lambda}\right) .
\end{aligned}
$$

But this suggests that $s_{2}\left(q_{t}, \bar{\lambda}\right)<s_{1}\left(q_{t}, \bar{\lambda}\right)$. Similarly,

$$
\begin{aligned}
& \int u^{\prime}\left(c_{1}\left(f\left(s_{1}\left(q_{t}, \underline{\lambda}\right)\right) \lambda_{t+1}, \lambda_{t+1}\right)\right) \lambda_{t+1} d G^{1}\left(\lambda_{t+1} ; \underline{\lambda}\right) \\
& \quad<\int u^{\prime}\left(c_{1}\left(f\left(s_{1}\left(q_{t}, \underline{\lambda}\right)\right) \lambda_{t+1}, \lambda_{t+1}\right)\right) \lambda_{t+1} d G^{2}\left(\lambda_{t+1} ; \underline{\lambda}\right),
\end{aligned}
$$


so that

$$
\begin{array}{r}
u^{\prime}\left(c_{1}\left(q_{t}, \underline{\lambda}\right)\right)<\beta f^{\prime}\left(s_{1}\left(q_{t}, \underline{\lambda}\right)\right) \int u^{\prime}\left(c _ { 1 } \left(f\left(s_{1}\left(q_{t}, \underline{\lambda}\right)\right)\right.\right. \\
\left.\left.\times \lambda_{t+1}, \lambda_{t+1}\right)\right) \lambda_{t+1} d G^{2}\left(\lambda_{t+1}, \underline{\lambda}\right),
\end{array}
$$

which implies $s_{1}\left(q_{t}, \underline{\lambda}\right)<s_{2}\left(q_{t}, \underline{\lambda}\right) \forall q_{t}$. Continuity considerations, together with these results (which could be duplicated for choices of random shocks distinct from $\underline{\lambda}$ and $\bar{\lambda}$ ), suggest the existence of some $\hat{\lambda}$ for which, $\forall q_{\text {t }}$

$$
\begin{array}{ll}
s_{2}\left(q_{t}, \lambda\right)>s_{1}\left(q_{t}, \lambda\right), & \underline{\lambda} \leqslant \lambda<\hat{\lambda}, \\
s_{2}\left(q_{t}, \lambda\right)<s_{1}\left(q_{t}, \lambda\right), & \bar{\lambda}<\lambda \leqslant \bar{\lambda} .
\end{array}
$$

This will have two effects, depending on agents' degree of relative risk aversion (see Theorem 3.1). If agents are highly risk averse (and thus $\left.\partial s\left(q, \lambda_{t}\right) / \partial \lambda_{t}<0\right)$ the following pattern might well be observed. (Fig. 8)

Agents' action thus reflect their altered expectations in a natural way. Under $G^{1}\left(\lambda_{t+1} ; \lambda_{t}\right)$, if the realization of the random shock were low, more (proportionately) would be saved; under $G^{2}\left(\lambda_{t+1} ; \lambda_{t}\right)$, since this low realization is believed more likely to persist, even more is saved. The corresponding phenomenon is observed for high realization.

Alternatively, if $\partial s\left(q, \lambda_{t}\right) / \partial \lambda_{t}>0$ (the case for less risk averse agents) we would observe (Fig. 9).

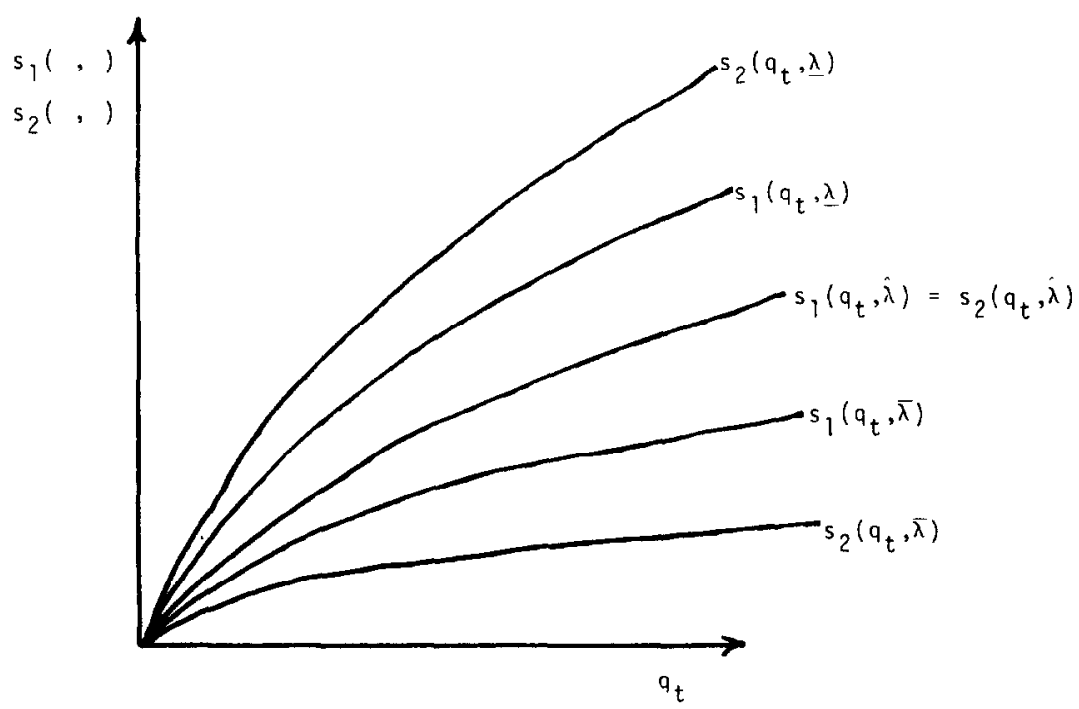

Figure 8 


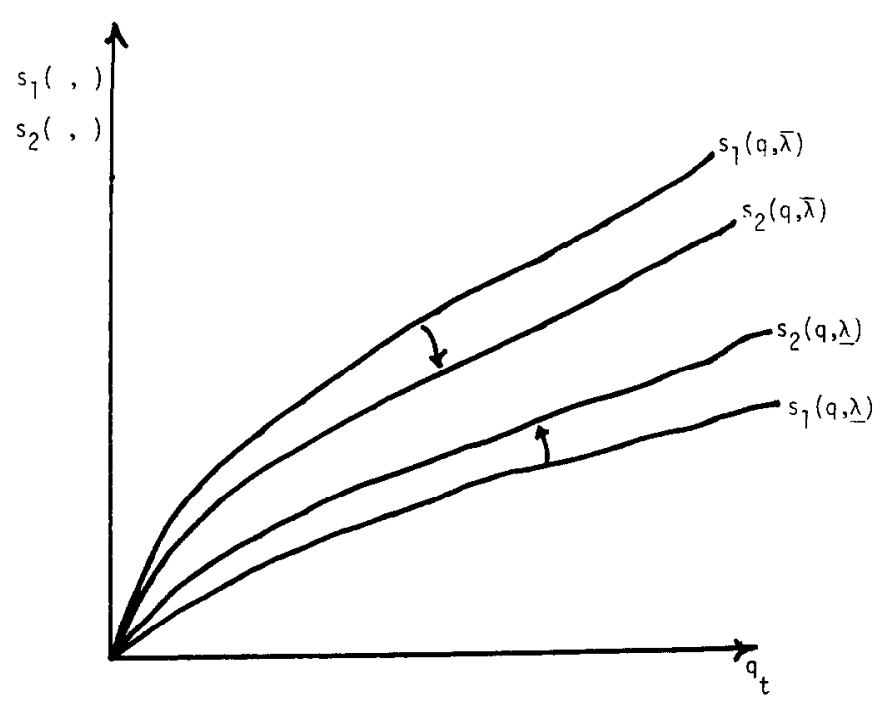

Figure 9

Dependent on agents' risk aversion, these diagrams thus suggest that such informational changes can increase or decrease the variability of the stationary distribution of capital stock (and thus also of consumption and output).

As can be easily checked, the effect of uniformly more favorable expectations is to reduce savings at all output levels, for all values of the random shock $\lambda$. The intuition for this result is clear.

\section{Concluding Comments}

The object of this paper has been to extend the neoclassical stochastic growth model by introducing correlated production shocks. The analysis provides a more general framework to incorporate explicitly the role of expectations. Current decisions of economic agents, especially savings and consumption decisions, are typically dependent upon expectations of future economic conditions.

In the context of the present model we can evaluate the effects of alternate expectational schemes on the stationary distributions of savings and consumption, in particular their means and variances. This has implications for policymaking since it provides the policy maker with a tool with which to "select" what expectations to induce in order to achieve a desired savings pattern. In particular one could test the impact of expectations induced by the various "constant policy rules" and see their effects on the stability of the 
economy. One could even simulate the dynamics of the economy as it converges to equilibrium.

Since it has been shown that the consumption-savings-investment policies of this economy are identical to those of a homogeneous consumer economy in recursive competitive equilibrium, one could examine the influence of expectations on risk premia and the risk structure of security prices. This will be discussed in a separate paper.

\section{REFERENCES}

1. F. BLACK, General equilibrium and business cycles, unpublished manuscript, Massachusetts Institute of Technology, 1979.

2. L. BLume, The ergodic behavior of stochastic processes of economic equilibria, Econometrica 47 (1979), 1421-1432.

3. L. Blume, D. EAsLey, AND M. O'HARA, Characterization of optimal plans for stochastic dynamic programming, Forthcoming J. Econ. Theory.

4. W. A. Brock, An integration of stochastic growth theory and the theory of finance, part 1: the growth model, in "General Equilibrium Growth and Trade" (J. Green and J. Scheinkman, Eds.), Academic Press, New York, 1979.

5. W. A. Brock and L. J. Mirman, Optimal economic growth and uncertainty: the discounted case, J. Econ. Theory 4 (1972), 497-513.

6. W. A. BROCK AND L. J. Mirman, Optimal economic growth and uncertainty: the no discounting case, Internat. Econ. Rev. 14 (1973), 560-573.

7. D. CAss, Optimal growth in an aggregative model of capital accumulation, Rev. Econ. Studies 32 (1965), 233-240.

8. J. P. Danthine and J. B. Donaldson, Stochastic properties of fast vs slow growing economies, Econometrica 49 (1981), 1007-1033.

9. J. P. Danthine AND J. B. Donaldson, Investment, stability and taxation in a long run macroeconomic model, unpublished manuscript, Columbia University, 1981.

10. A. Dixit, "Theory of Equilibrium Growth," Oxford Univ. Press, London, 1976.

11. J. Drèzé and F. Modigliani, Consumption decisions under uncertainty, J. Econ. Theory 5 (1972), 308-335.

12. W. Feller, "An Introduction to Probability Theory and Its Application," Wiley, New York, 1966.

13. F. H. HAHN, Savings and uncertainty, Rev. Econ. Studies 37 (1970), 21-24.

14. M. HARRIS, Note sets for dynamic competitive analysis, unpublished manuscript, Carnegie-Mellon University, 1978.

15. T, Koopmans, On the concept of optimal economic growth, in "Semaine d'Etude sur le Rôle de l'Analysis Econometrique dans la Formulation de plans de Development," Pontifican Academiae Scientiarium Scripta Varia, No. 28, Vatican, 1965.

16. F. Kydland and E. C. Prescott, Time to build and the persistence of unemployment, Econometrica 50 (1982), 1345-1370.

17. D. Levhari and T. SRINIvasan, Optimal savings under uncertainty, Rev. Econ. Studies 36 (1969), 153-164.

18. R. E. LuCAs, An equilibrium model of the business cycle, J. Pol. Econ. 83 (1975), 1113-1144.

19. R. E. LuCAs AND E. C. Prescott, Investment under uncertainty, Econometrica 39 (1971) 659-681. 
20. L. W. MCKENZIE, On the existence of general equilibrium for a competitive market, Econometrica 27 (1959), 54-71.

21. R. Mendelssohn and M. Sobel, Capital accumulation and the optimization of renewable resource models, J. Econ. Theory 23 (1980), 243-260.

22. R. Merton, An asymptotic theory of growth under uncertainty, Rev. Econ. Studies 42 (1975), 375-393.

23. L. J. MiRman AND I. Zilcha, On optimal growth under uncertainty, J. Econ. Theory 11 (1975), 329-339.

24. L. J. Mirman and I. Zilcha, Unbounded shadow prices for optimal stochastic growth models, Internat. Econ. Rev. 17 (1976), 121-132.

25. J. A. MirRLeES, Optimal accumulation under uncertainty: the case of stationary returns of investment, in "Allocation under Uncertainty: Equilibrium and Optimality," (J. Drèzé, Ed.), Wiley, New York, 1974.

26. E. S. Phelps, The accumulation of risky capital: A sequential utility analysis, Econometrica 30 (1962), 729-743.

27. E. C. Prescott and R. MEhra, Recursive competitive equilibrium: The case of homogeneous households, Econometrica 48 (1980), 1365-1379.

28. M. RothSCHILD AND J. E. STIGLITZ, Increasing risk 11: Its economic consequences, $J$. Econ. Theory 4 (1971), 66-84. 